\title{
HOMOMORPHISMS INTO SIMPLE LIMITS OF CIRCLE ALGEBRAS
}

\author{
KAREN EGEDE NIELSEN*
}

\section{Introduction}

Given any class of $C^{*}$-algebras it is an important problem to describe the nature of the $*$-homomorphisms connecting the $C^{*}$-algebras. Indeed, if one can prove that there is a 1-1 correspondence, up to approximate inner equivalence, between the $*$-homomorphisms connecting any two algebras in the class and the morphisms connecting some natural invariants of the two $C^{*}$-algebras, then (as long as the $C^{*}$-algebras are separable) this automatically yields a classification of the $C^{*}$-algebras in terms of the same invariants, cf. [19].

For a unital $C^{*}$-algebra, $A$, the so-called Elliott Invariant is the tuple consisting of the $\mathrm{K}$-groups, $\mathrm{K}_{0}(A) \oplus \mathrm{K}_{1}(A)$, the tracial state space, $T(A)$, and the pairing map, $r_{A}$. Elliott's classification theorem, Theorem 1 of [10], says that this invariant is complete for the unital $C^{*}$-algebras which arise as inductive limits of sequences of finite direct sums of circle algebras (for short this class of $C^{*}$-algebras will be denoted by $\mathscr{C}_{\mathrm{T}}$ ) and which are simple. In [16], this was clarified by showing that in fact any morphism at the level of the Elliott Invariants lift to a $*$-homomorphism at the level of the $C^{*}$-algebras and further, that this lift is uniquely determined, up to approximate inner equivalence, by its action on a natural extension of the Elliott Invariant.

The purpose of this paper is, as a step towards a better understanding of the non-simple case, to describe the nature of the unital $*$-homomorphisms from an arbitrary $C^{*}$-algebra from $\mathscr{C}_{\mathrm{T}}$ into a simple $C^{*}$-algebra from $\mathscr{C}_{\mathrm{T}}$. In particular, we focus on the existence and uniqueness of $*$-homomorphisms lifted from morphisms between the Elliott Invariants of the algebras.

We show that given a unital $*$-homomorphisms between two $C^{*}$-algebras from $\mathscr{C}_{\mathrm{T}}$, only the target algebra being simple, then the $*$-homomorphism is

\footnotetext{
* Supported by "Rejselegat for Matematikere".

Received August 22, 1996.
} 
completely determined, up to approximate inner equivalence, by its action at the level of the extended invariant from [16]. This is Theorem 2.1 below.

Contrary to the case where both the target and the domain algebra are simple, in our case, we have natural obstructions against the lifting of some morphisms between the invariants. These obstructions are discussed, and we describe exactly which morphism between the Elliott Invariants (as well as between the extended invariants from [16]) that can be lifted to *-homomorphisms between the algebras. For details see Theorem 3.3 below.

For $C^{*}$-algebras in $\mathscr{C}_{\top}$ of real rank zero, the Elliott Invariant reduces to the K-groups of the algebras and, by [8], any positive, order unit preserving map between the $\mathrm{K}$-groups of two such algebras lift to a $*$-homomorphism between the $C^{*}$-algebras. In section 4 we show that not all positive, order unit preserving maps between the K-groups of two simple $C^{*}$-algebras in $\mathscr{C}_{\mathrm{T}}$ lift to $*$-homomorphisms .

Finally, by applying our results to the special case where the domain algebra is $C(\mathrm{~T})$, we obtain a classification, up to approximate unitary equivalence, of the unitary elements in simple $C^{*}$-algebras from $\mathscr{C}_{\mathrm{T}}$. For details see Theorem 5.1 below.

The author wish to thank the referee for shortening the proof of Lemma 2.2 .

\section{Preliminaries}

Let $\mathscr{C}_{\mathrm{T}}$ denote the class of all unital $C^{*}$-algebras which are inductive limits of sequences of finite direct sums of circle algebras. If $A$ in $\mathscr{C}_{\mathrm{T}}$ is simple and infinite dimensional, it can be realized as an inductive limit of a sequence of finite direct sums of circle algebras with injective connecting $*$-homomorphisms (Theorem 1.1 of [16]). Throughout, when dealing with a simple infinite dimensional $C^{*}$-algebra in $\mathscr{C}_{\mathrm{T}}$, we will assume that it has been realized in this manner.

When $A$ is a unital $C^{*}$-algebra, $U(A)$ will denote the unitary group and $\overline{D U(A)}$ the closure of its commutator subgroup. Then $U(A) / \overline{D U(A)}$ is a metrizable complete topological group in the quotient metric

$$
D_{A}(Q(u), Q(v))=\inf \left\{\left\|u v^{*}-c\right\| \mid c \in \overline{D U(A)}\right\},
$$

where $Q: U(A) \rightarrow U(A) / \overline{D U(A)}$ denote the quotient map. We let $\rho: \mathrm{K}_{0}(A) \rightarrow \operatorname{Aff} T(A)$ denote the natural map and $q:$ Aff $T(A) \rightarrow$ Aff $T(A) / \overline{\rho\left(\mathrm{K}_{0}(A)\right)}$ the induced quotient map. If $d^{\prime}$ denotes the quotient metric on Aff $T(A) / \overline{\rho\left(\mathrm{K}_{0}(A)\right)}$, the metric $d_{A}$ on Aff $T(A) / \overline{\rho\left(\mathrm{K}_{0}(A)\right)}$, defined by 


$$
d_{A}(q(f), q(g))= \begin{cases}2 & \text { for } d^{\prime}(q(f), q(g)) \geq \frac{1}{2} \\ \left|e^{2 \pi i d^{\prime}(q(f), q(g))}-1\right| & \text { for } d^{\prime}(q(f), q(g))<\frac{1}{2}\end{cases}
$$

also induces the quotient topology on Aff $T(A) / \overline{\rho\left(\mathrm{K}_{0}(A)\right)}$. Given $a=\left(a_{i j}\right) \in M_{k}(A)_{\mathrm{sa}}$ for some $k \in \mathrm{N}$, then $\widehat{a} \in$ Aff $T(A)$ will denote the element $\omega \mapsto \sum_{i=1}^{k} \omega\left(a_{i i}\right), \omega \in T(A)$. When $A \in \mathscr{C}_{\mathrm{T}}, \quad \overline{D U(A)} \subseteq U_{0}(A)$, $\mathrm{K}_{1}(A) \simeq \pi_{0}(U(A)), \mathrm{K}_{0}(A) \simeq \pi_{1}(U(A))$, cf. [18], and the map

$$
\lambda_{A}: \text { Aff } T(A) / \overline{\rho\left(\mathrm{K}_{0}(A)\right)} \rightarrow U(A) / \overline{D U(A)} ; \lambda_{A}(\widehat{a})=Q\left(e^{2 \pi i a}\right) \quad \forall a \in A_{\mathrm{sa}},
$$

is a well-defined embedding, which identifies Aff $T(A) / \overline{\rho\left(\mathrm{K}_{0}(A)\right)}$ with $U_{0}(A) / \overline{D U(A)}$, cf. [20] and [16]. It follows that we have an exact sequence

$$
0 \rightarrow \operatorname{Aff} T(A) / \overline{\rho\left(\mathrm{K}_{0}(A)\right)} \stackrel{\lambda_{A}}{\longrightarrow} U(A) / \overline{D U(A)} \stackrel{\pi_{A}}{\longrightarrow} \mathrm{K}_{1}(A) \rightarrow 0
$$

where $\pi_{A}(Q(u))=[u]_{\mathrm{K}_{1}}, u \in U(A)$. Moreover, by Lemma 3.1 of [16], this sequence is split exact and $\lambda_{A}$ is an isometry with respect to the metrics $d_{A}$ and $D_{A}$.

A unital $*$-homomorphism $\varphi: A \rightarrow B$ between unital $C^{*}$-algebras induces in a natural way a contractive homomorphism $\varphi^{\natural}: U(A) / \overline{D U(A)} \rightarrow$ $U(B) / \overline{D U(B)}$ between the unitary groups modulo the closure of their commutator subgroups. Moreover, when $A$ and $B$ are from the class $\mathscr{C}_{\mathrm{T}}$, the action of $\varphi$ at the level of $\mathrm{K}_{1}$ can be recovered from $\varphi^{\natural}$ as the map between the groups of connected components.

Let $A=\lim \left\{A_{n}, \mu_{n}\right\}$ be an inductive limit of a sequence of finite direct sums of circle algebras. Then (cf. Lemma 3.2 and Lemma 3.3 of [21]) the order unit space Aff $T(A)$ is the inductive limit

$$
\text { Aff } T\left(A_{1}\right) \stackrel{\widehat{\mu_{1}}}{\longrightarrow} \text { Aff } T\left(A_{2}\right) \stackrel{\widehat{\mu_{2}}}{\longrightarrow} \text { Aff } T\left(A_{3}\right) \stackrel{\widehat{\mu_{3}}}{\longrightarrow} \cdots
$$

and the canonical map Aff $T\left(A_{n}\right) \rightarrow$ Aff $T(A)$ is the map $\widehat{\mu_{\infty, n}}$. Similarly, $U(A) / \overline{D U(A)}$ is the inductive limit, in the category of complete metric groups, of the sequence

$$
U\left(A_{1}\right) / \overline{D U\left(A_{1}\right)} \stackrel{\mu_{1}^{\natural}}{\longrightarrow} U\left(A_{2}\right) / \overline{D U\left(A_{2}\right)} \stackrel{\mu_{2}^{\natural}}{\longrightarrow} U\left(A_{3}\right) / \overline{D U\left(A_{3}\right)} \stackrel{\mu_{3}^{\natural}}{\longrightarrow} \cdots
$$

where the canonical map $U\left(A_{n}\right) / \overline{D U\left(A_{n}\right)} \rightarrow U(A) / \overline{D U(A)}$ is the map $\mu_{\infty, n}^{\natural}$. This will be used freely in the following.

\section{Uniqueness}

We will prove the following theorem, which is the counterpart of [16] Theorem $\mathrm{B}$.

Theorem 2.1. Given $A, B \in \mathscr{C}_{\top}$ with $B$ simple. Let $\varphi, \psi: A \rightarrow B$ be two 
unital $*$-homomorphisms. Then $\varphi$ and $\psi$ are approximate inner equivalent if and only if $\varphi_{*}=\psi_{*}$ on $\mathrm{K}_{0}(A), \varphi^{*}=\psi^{*}$ on $T(B)$ and $\varphi^{\natural}=\psi^{\natural}$ on $U(A) / \overline{D U(A)}$.

It is possible to give an example of a simple unital $C^{*}$-algebra in $\mathscr{C}_{\mathrm{T}}$ which has an automorphism $\alpha$ that is not approximately inner, although $\alpha$ has the same action as the identity map on the K-theory and the tracial state space cf. section 5 of [16]. Thus, in general, it does not suffice in Theorem 2.1 that the $*$-homomorphisms $\varphi$ and $\psi$ have the same action on the $\mathrm{K}$-theory and the tracial state spaces. To prove the theorem we will isolate two lemmas.

Lemma 2.2. Let $A, B$ be unital $C^{*}$-algebras with $B$ simple and $T(B) \neq \emptyset$. Let $\varphi, \psi: A \rightarrow B$ be two unital $*$-homomorphisms. If $\varphi^{*}=\psi^{*}$ on $T(B)$, then $\operatorname{ker} \varphi=\operatorname{ker} \psi$.

Proof. Given $a \in A$, then by assumption $\tau\left(\varphi\left(a^{*} a\right)\right)=\tau\left(\psi\left(a^{*} a\right)\right)$ for all $\tau \in T(B)$. Since all traces on $B$ are faithful ( $B$ is simple) it follows that $\varphi(a)=0$ if and only if $\psi(a)=0$.

Lemma 2.3 below is an adaptation of Elliott's uniqueness theorem for $*$ homomorphisms between finite direct sums of interval algebras, Theorem 6 of [9], to the case of $*$-homomorphisms from finite direct sums of interval algebras to finite direct sums of circle algebras.

Given $A=\oplus_{j=1}^{K} C(X) \otimes M_{n_{j}}$ a finite direct sum of circle (i.e. $X=\mathrm{T}$ ), respectively interval algebras (i.e. $X=[0,1]$ ). The canonical generators $\operatorname{cg}(A)$ of $A$ consist of the standard system of matrix units of $\oplus_{j=1}^{K} M_{n_{j}}$ together with the unitary, respectively selfadjoint element (id, id, $\cdots, \mathrm{id}$ ). When $A$ is a finite direct sum of interval algebras, we let $c a_{0}(A)$ be the set consisting of the images in $A$ of the canonical selfadjoint generator of the center of each of the summands $C([0,1]) \otimes M_{n_{j}}, j=1, \cdots, K$. When $A$ is a finite direct sum of circle algebras, $\mathrm{cu}(A)$ denotes the set consisting of the unitaries (id, $1, \cdots, 1),(1, \mathrm{id}, 1, \cdots, 1), \cdots,(1, \cdots, 1, \mathrm{id})$, whereas the set consisting of the partial unitaries $(\mathrm{id}, 0, \cdots, 0),(0$, id, $0, \cdots, 0), \cdots,(0, \cdots, 0$, id $)$ will be denoted by $\operatorname{cu}_{0}(A)$. Given $n \in \mathrm{N}$ let $\zeta_{i}:[0,1] \rightarrow[0,1], i=1, \cdots, n$, be non-zero continuous functions such that $\operatorname{supp} \zeta_{i} \subseteq\left[\frac{i-1}{n}, \frac{i}{n}\right]$.

Lemma 2.3. For every $n \in \mathrm{N}$ there is a finite set of functions $G \subseteq C[0,1]$ with the following property: When $A$ is a finite direct sum of interval algebras, $B$ a finite direct sum of circle algebras, $\varphi, \psi: A \rightarrow B$ unital $*$-homomorphisms and $\delta>0$ such that

(1) $\varphi_{*}=\psi_{*}$ on $\mathrm{K}_{0}(A)$,

(2) $\theta\left(\zeta_{i}\left(\varphi\left(a_{0}\right)\right)\right)>2 \delta \forall i=1, \cdots, n, \theta \in T(B), a_{0} \in \mathrm{ca}_{0}(A)$,

(3) $\left|\theta\left(\varphi\left(g\left(a_{0}\right)\right)\right)-\theta\left(\psi\left(g\left(a_{0}\right)\right)\right)\right|<\delta \forall g \in G, \theta \in T(B), a_{0} \in \operatorname{ca}_{0}(A)$.

Then there is a unitary $u \in U(B)$ such that 


$$
\left\|u \varphi(a) u^{*}-\psi(a)\right\| \leq \frac{3}{n}, \quad a \in \operatorname{cg}(A) .
$$

Proof. It is straightforward to adapt the proof of Theorem 6 of [9] to the present situation.

Proof of Theorem 2.1. Let $\left\{A_{n}, \mu_{n}\right\}$ and $\left\{B_{n}, \rho_{n}\right\}$ be generating sequences of $A$ and $B$, respectively. It suffices to find a sequence of unitaries $\left\{u_{k}\right\}$ in $B$ such that

(1) $\left\|u_{k} \varphi \circ \mu_{\infty, k}(a) u_{k}^{*}-\psi \circ \mu_{\infty, k}(a)\right\|<\frac{1}{k} \quad \forall a \in \bigcup_{j=1}^{k-1} \mu_{k, j}\left(\operatorname{cg}\left(A_{j}\right)\right) \cup \operatorname{cg}\left(A_{k}\right)$.

Let $k \in \mathrm{N}$ be given. By Lemma 2.2, ker $\varphi \circ \mu_{\infty, k}=\operatorname{ker} \psi \circ \mu_{\infty, k}$. There are closed subsets $X_{1}, \cdots, X_{M}$ of $\mathrm{T}$ such that $A_{k} / \operatorname{ker} \varphi \circ \mu_{\infty, k} \simeq$ $\oplus_{j=1}^{M} C\left(X_{j}\right) \otimes M_{n_{j}}$. From Lemma 1.3 of [16] it follows that for any finite subset $F \subseteq \oplus_{j=1}^{M} C\left(X_{j}\right) \otimes M_{n_{j}}$ and any $\epsilon>0$ there exist regular subsets $Y_{j} \subseteq X_{j}, j=1, \cdots, M$, and an injective $*$-homomorphism

$$
\phi: \oplus_{j=1}^{M} C\left(Y_{j}\right) \otimes M_{n_{j}} \rightarrow \oplus_{j=1}^{M} C\left(X_{j}\right) \otimes M_{n_{j}}
$$

such that

$$
F \subseteq_{\epsilon} \phi\left(\oplus_{j=1}^{M} C\left(Y_{j}\right) \otimes M_{n_{j}}\right)
$$

By a regular subset of $\mathrm{T}$ we mean a subset which is either $\mathrm{T}$ or the union of finitely many points and closed arc-segments. It follows that there exist a $\delta_{0}>0$ and regular subsets $Y_{j} \subseteq X_{j}, j=1, \cdots, M$, such that the estimate (1) will follow, if we can prove that

$$
\left\|u_{k} \varphi \circ \mu_{\infty, k} \circ \phi(a) u_{k}^{*}-\psi \circ \mu_{\infty, k} \circ \phi(a)\right\|<\delta_{0}, a \in \operatorname{cg}\left(A_{k}^{\prime}\right),
$$

where $A_{k}^{\prime}=\oplus_{j=1}^{M} C\left(Y_{j}\right) \otimes M_{n_{j}}$.

$A_{k}^{\prime}$ is isomorphic to a finite direct sum of algebras, each summand being either an interval-, a circle- or a matrix-algebra. However, using that $\varphi_{*} \circ \mu_{\infty, k_{*}} \circ \phi_{*}=\psi_{*} \circ \mu_{\infty, k_{*}} \circ \phi_{*}$ on $\mathrm{K}_{0}\left(A_{k}^{\prime}\right)$, we can treat each summand separately. In particular we can reduce to the following three cases - the case (i) where $A_{k}^{\prime}$ is a finite direct sum of interval algebras, the case (ii) where $A_{k}^{\prime}$ is a finite direct sum of circle algebras and the case (iii) where $A_{k}^{\prime}$ is finite dimensional. In the latter case, the theorem follows from the well-known fact that two unital $*$-homomorphisms from a finite dimensional $C^{*}$-algebra into a unital $C^{*}$-algebra with cancellation in $\mathrm{K}_{0}$ are inner equivalent. So what is left is case (i) and (ii);

Case (i): Adopt the notation from Lemma 2.3. Choose $n \in \mathrm{N}$ such that $\frac{3}{n}<\delta_{0}$. Let $G \subseteq C[0,1]$ be the finite set of functions from Lemma 2.3 corre- 
sponding to the choice of $n$. Since $B$ is simple and $\varphi \circ \mu_{\infty, k} \circ \phi$ is injective, we can find $\delta>0$ such that

$$
\theta\left(\zeta_{i}\left(\varphi \circ \mu_{\infty, k} \circ \phi\left(a_{0}\right)\right)\right)>2 \delta, i=1, \cdots, n, \theta \in T(B), a_{0} \in \mathrm{ca}_{0}\left(A_{k}^{\prime}\right) .
$$

Now for any finite subset $H \subseteq A_{k}^{\prime}$ and $\epsilon>0$ we can find an $\ell \in \mathrm{N}$ and unital *-homomorphisms $\varphi_{\ell}, \psi_{\ell}: A_{k}^{\prime} \rightarrow B_{\ell}$ such that $\| \rho_{\infty, \ell} \circ \varphi_{\ell}(a)-\varphi \circ \mu_{\infty, k} \circ$ $\phi(a) \|<\epsilon$ and $\left\|\rho_{\infty, \ell} \circ \psi_{\ell}(a)-\psi \circ \mu_{\infty, k} \circ \phi(a)\right\|<\epsilon$ for all $a \in H$, cf. Lemma 4.2 of [8]. Since $\widehat{\varphi} \circ \widehat{\mu_{\infty, k}} \circ \widehat{\phi}=\widehat{\psi} \circ \widehat{\mu_{\infty, k}} \circ \widehat{\phi} \quad$ on $\operatorname{Aff} T\left(A_{k}^{\prime}\right)$ and $\varphi_{*} \circ \mu_{\infty, k_{*}} \circ \phi_{*}=\psi_{*} \circ \mu_{\infty, k_{*}} \circ \phi_{*}$ on $\mathrm{K}_{0}\left(A_{k}^{\prime}\right)$, we can, by choosing $\epsilon$ and $H$ appropriately, arrange that

$$
\left\|\widehat{\varphi}_{\ell}\left(g \widehat{\left(a_{0}\right)}\right)-\widehat{\psi}_{\ell}\left(\widehat{g\left(a_{0}\right)}\right)\right\|<\delta, g \in G, a_{0} \in \mathrm{ca}_{0}\left(A_{k}^{\prime}\right),
$$

and, by (2),

$$
\theta\left(\zeta_{i}\left(\varphi_{\ell}\left(a_{0}\right)\right)\right)>2 \delta, i=1, \cdots, n, \theta \in T\left(B_{\ell}\right), a_{0} \in \mathrm{ca}_{0}\left(A_{k}^{\prime}\right),
$$

and further that $\varphi_{\ell_{*}}=\psi_{\ell_{*}}$ on $\mathrm{K}_{0}\left(A_{k}^{\prime}\right)$. Applying Lemma 2.3 we obtain a unitary $v_{k} \in B_{\ell}$ such that

$$
\left\|v_{k} \varphi_{\ell}(a) v_{k}^{*}-\psi_{\ell}(a)\right\| \leq \frac{3}{n}, a \in \operatorname{cg}\left(A_{k}^{\prime}\right) .
$$

Now, if $\epsilon$ is chosen smaller than $\frac{1}{2}\left(\delta_{0}-\frac{3}{n}\right)$ and if $H \supseteq \operatorname{cg}\left(A_{k}^{\prime}\right)$, then $u_{k}=\rho_{\infty, \ell}\left(v_{k}\right)$ is the desired unitary.

Case (ii): Adopt the notation from Theorem 2.4 of [16] (the uniqueness theorem for $*$-homomorphisms between finite direct sums of circle algebras). Choose $m \in \mathrm{N}$ such that $\frac{28 \pi}{m}<\frac{\delta_{0}}{2}$. Since $B$ is simple, we can find $n \in \mathrm{N}, n>12$ and $\delta>0$ such that $\frac{6 \pi}{n}<\frac{\delta_{0}}{2}$,

$$
\theta\left(\xi_{j}^{m}\left(\varphi \circ \mu_{\infty, k} \circ \phi\left(u_{0}\right)\right)\right)>\frac{1}{n}, j=1,2, \cdots, m, \theta \in T(B), u_{0} \in \mathrm{cu}_{0}\left(A_{k}^{\prime}\right),
$$

and

(4) $\theta\left(\xi_{i}^{3 n}\left(\varphi \circ \mu_{\infty, k} \circ \phi\left(u_{0}\right)\right)\right)>2 \delta, \quad i=1,2, \cdots, 3 n, \theta \in T(B), u_{0} \in \operatorname{cu}_{0}\left(A_{k}^{\prime}\right)$.

Let $F \subset C(\mathrm{~T} \cup\{0\},[0,1])$ be the finite set of functions from Theorem 2.4 of [16] corresponding to the present choice of $n$ and $m$. Analogously to case (i), we can find $\ell \in \mathrm{N}$ and $*$-homomorphisms $\varphi_{\ell}, \psi_{\ell}: A_{k}^{\prime} \rightarrow B_{\ell}$ such that $\varphi_{\ell *}=\psi_{\ell *}$ on $\mathrm{K}_{0}\left(A_{k}^{\prime}\right)$,

$$
\max \left\{\left\|\rho_{\infty, \ell} \circ \varphi_{\ell}(a)-\varphi \circ \mu_{\infty, k} \circ \phi(a)\right\|,\left\|\rho_{\infty, \ell} \circ \psi_{\ell}(a)-\psi \circ \mu_{\infty, k} \circ \phi(a)\right\|\right\}
$$




$$
<\frac{1}{2}\left(\delta_{0}-\left(\frac{28 \pi}{m}+\frac{6 \pi}{n}\right)\right), a \in \operatorname{cg}\left(A_{k}^{\prime}\right)
$$

and

$$
\left\|\widehat{\varphi}_{\ell}\left(\widehat{f\left(u_{0}\right)}\right)-\widehat{\psi_{\ell}}\left(\widehat{f\left(u_{0}\right)}\right)\right\|<\delta, \quad f \in F, u_{0} \in \operatorname{cu}_{0}\left(A_{k}^{\prime}\right) .
$$

We can also arrange that

$$
\operatorname{dist}\left(\psi_{\ell}(u) \varphi_{\ell}\left(u^{*}\right), D U\left(B_{\ell}\right)\right)<\frac{1}{n^{2}}, \quad u \in \operatorname{cu}\left(A_{k}^{\prime}\right)
$$

since $\varphi^{\natural} \circ \mu_{\infty, k}^{\natural} \circ \phi^{\natural}=\psi^{\natural} \circ \mu_{\infty, k}^{\natural} \circ \phi^{\natural}$ on $U\left(A_{k}^{\prime}\right) / \overline{D U\left(A_{k}^{\prime}\right)}$, and further, by (3) and (4), we can arrange that

$$
\theta\left(\varphi_{\ell}\left(\xi_{j}^{m}\left(u_{0}\right)\right)\right)>\frac{1}{n}, \quad j=1,2, \cdots, m, \theta \in T\left(B_{\ell}\right), u_{0} \in \mathrm{cu}_{0}\left(A_{k}^{\prime}\right)
$$

and

$$
\theta\left(\varphi_{\ell}\left(\xi_{i}^{3 n}\left(u_{0}\right)\right)\right)>2 \delta, \quad i=1,2, \cdots, 3 n, \theta \in T\left(B_{\ell}\right), u_{0} \in \operatorname{cu}_{0}\left(A_{k}^{\prime}\right) .
$$

Now the desired unitary can be obtained from Theorem 2.4 of [16].

\section{Existence}

We discuss the natural obstructions against the lifting of a map at the level of the invariants to a $*$-homomorphisms at the level of the $C^{*}$-algebras, the domain algebra and the target algebra being an arbitrary and a simple $C^{*}$ algebra from the class $\mathscr{C}_{\mathrm{T}}$, respectively. Furthermore, we show that the obstructions described are the only ones by proving an existence theorem, Theorem 3.3 below, for unital $*$-homomorphisms from an arbitrary into a simple $C^{*}$-algebra from the class $\mathscr{C}_{\mathrm{T}}$.

Let $A, B \in \mathscr{C}_{\mathrm{T}}$. If $\varphi_{0}: \mathrm{K}_{0}(A) \rightarrow \mathrm{K}_{0}(B)$ is a positive homomorphism, $\varphi_{T}: T(B) \rightarrow T(A)$ a continuous, affine map and $\Psi: U(A) / \overline{D U(A)} \rightarrow$ $U(B) / \overline{D U(B)}$ a group homomorphism, then we will say that the tuple $\left(\varphi_{0}, \varphi_{T}, \Psi\right)$ is compatible when

$$
r_{A} \circ \varphi_{T}(\omega)(x)=r_{B}(\omega)\left(\varphi_{0}(x)\right), \quad x \in \mathrm{K}_{0}(A), \omega \in T(B),
$$

and

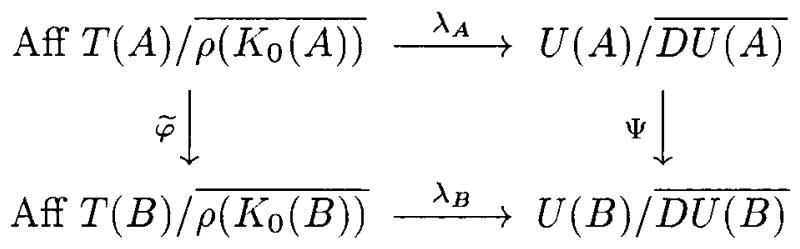


commutes. Here $r_{(\cdot)}: T(\cdot) \rightarrow S\left(\mathrm{~K}_{0}(\cdot)\right)$ denotes the pairing map and $\widetilde{\varphi}:$ Aff $T(A) \overline{\rho\left(\mathrm{K}_{0}(A)\right)} \rightarrow$ Aff $T(B) / \overline{\rho\left(\mathrm{K}_{0}(B)\right)}$ the homomorphism induced by $\varphi_{0}$ and $\varphi_{T}$. Let $\mathbf{E}(A)$ denote the following extension of the Elliott Invariant

$$
\mathbf{E}(A)=\left(\mathrm{K}_{0}(A), T(A), U(A) / \overline{D U(A)}, r_{A}, \lambda_{A}\right) .
$$

Then a compatible tuple can be considered as a morphism $\mathbf{E}(A) \rightarrow \mathbf{E}(B)$.

When $\varphi_{0}: \mathrm{K}_{0}(A) \rightarrow \mathrm{K}_{0}(B)$ is a positive homomorphism and $\varphi_{T}: T(B) \rightarrow$ $T(A)$ a continuous, affine map, compatible with $\varphi_{0}$. Then the map $\widetilde{\varphi}:$ Aff $T(A) / \overline{\rho\left(\mathrm{K}_{0}(A)\right)} \rightarrow$ Aff $T(B) / \overline{\rho\left(\mathrm{K}_{0}(B)\right)}$ is contractive w.r.t. the metrics $d_{A}$ and $d_{B}$. Let $\Psi: U(A) / \overline{D U(A)} \rightarrow U(B) / \overline{D U(B)}$ be a group homomorphisms for which the first square of the diagram

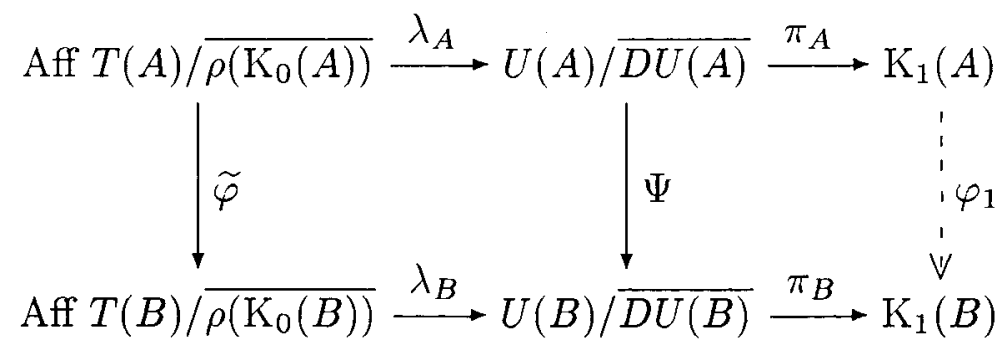

commutes. Then, because $\lambda_{A}$ and $\lambda_{B}$ are isometries, it follows that $\Psi$ is contractive. Further, since $\mathrm{K}_{1}(A)$ and $\mathrm{K}_{1}(B)$ are the groups of connected components in $U(A) / \overline{D U(A)}$ and $U(B) / \overline{D U(B)}$, respectively, it follows that $\Psi$ induces a group homomorphism $\varphi_{1}: \mathrm{K}_{1}(A) \rightarrow \mathrm{K}_{1}(B)$ such that the entire diagram is commutative. Suppressing the $*$-isomorphisms arising from the split exactness of the rows, $U(A) / \overline{D U(A)} \simeq \operatorname{Aff} T(A) / \overline{\rho\left(\mathrm{K}_{0}(A)\right)} \oplus \mathrm{K}_{1}(A)$ and $U(B) / \overline{D U(B)} \simeq$ Aff $T(B) / \overline{\rho\left(\mathrm{K}_{0}(B)\right)} \oplus \mathrm{K}_{1}(B), \Psi$ decomposes into four homomorphisms

$$
\left(\begin{array}{ll}
\Psi_{11} & \Psi_{12} \\
\Psi_{21} & \Psi_{22}
\end{array}\right)
$$

where $\Psi_{11}=\widetilde{\varphi}, \Psi_{21}=0, \Psi_{22}=\varphi_{1}$ and $\Psi_{12}$ can be any homomorphism $\mathrm{K}_{1}(A) \rightarrow$ Aff $T(B) / \overline{\rho\left(\mathrm{K}_{0}(B)\right)}$. Conversely, any homomorphism of this form makes the entire diagram commutative. It follows that if $\mathrm{E}(A)$ denotes the Elliott Invariant, i.e.

$$
\mathrm{E}(A)=\left(\mathrm{K}_{0}(A), T(A), \mathrm{K}_{1}(A), r_{A}\right),
$$

then $\mathbf{E}(A)$ is a natural extension of $\mathrm{E}(A)$ in the sense that any morphism $\mathbf{E}(A) \rightarrow \mathbf{E}(B)$ restricts to a morphism $\mathrm{E}(A) \rightarrow \mathrm{E}(B)$ and, conversely, any 
morphism $\mathrm{E}(A) \rightarrow \mathrm{E}(B)$ extends (although not necessarily in a unique way) to a morphism $\mathbf{E}(A) \rightarrow \mathbf{E}(B)$.

Let $A, B$ be unital $C^{*}$-algebras. By the tracial scale of $A$ we will mean the cone

$$
\Sigma^{t}(A)=\left\{\widehat{a^{*} a} \in \operatorname{Aff} T(A) \mid a \in A\right\} \subseteq \operatorname{Aff} T(A)^{+} .
$$

A continuous affine map $\varphi_{T}: T(B) \rightarrow T(A)$ will be said to be scale preserving when its dual map $\varphi_{T *}$ satisfies that $\varphi_{T *}\left(\Sigma^{t}(A)\right) \subseteq \Sigma^{t}(B)$. From [5] it follows that for any unital $C^{*}$-algebra $D,\{f \in \operatorname{Aff} T(D) \mid f>0\} \subseteq \Sigma^{t}(D)$. Using this, it is easy to see that when $A, B$ are unital $C^{*}$-algebras and $A$ is simple, then any continuous, affine map $\varphi_{T}: T(B) \rightarrow T(A)$ is scale preserving. When $A$ is not simple, however, this is no longer the case, cf. Example 3.6.

Given a continuous, affine map $\varphi_{T}: T(B) \rightarrow T(A)$, we let $I_{\varphi_{T}} \subseteq A$ denote the closed two-sided ideal

$$
I_{\varphi_{T}}=\left\{a \in A \mid \varphi_{T_{*}}\left(\widehat{a^{*} a}\right)=0\right\},
$$

and let $\pi: A \rightarrow A / I_{\varphi_{T}}$ denote the quotient map.

Lemma 3.1. Let $A, B$ be unital $C^{*}$-algebras. Given a continuous, affine map $\varphi_{T}: T(B) \rightarrow T(A)$, there exists a unique Markov operator $\phi:$ Aff $T\left(A / I_{\varphi_{T}}\right) \rightarrow$ Aff $T(B)$ such that the following diagram

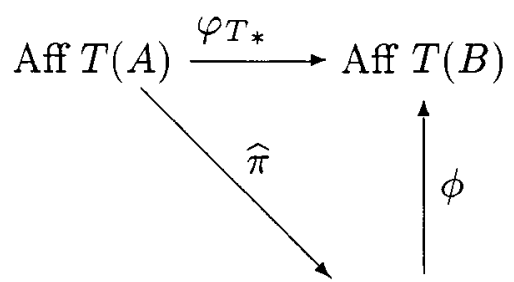

Aff $T\left(A / I_{\varphi_{T}}\right)$

is commutative. Moreover, $\phi$ is faithful, and if $\varphi_{T}$ is scale preserving, then so is $\phi$.

Proof. For any unital $C^{*}$-algebra $D$, let $D_{0} \subseteq D_{\text {sa }}$ denote the subset of elements $x-y, x, y \in D_{\text {sa }}$, for which there exists a sequence $\left\{d_{i}\right\} \subseteq D$ with $x=\sum_{i} d_{i} d_{i}^{*}$ and $y=\sum_{i} d_{i}^{*} d_{i}$. Then $D_{0} \subseteq D_{\mathrm{sa}}$ is closed, and for all $d \in D_{\mathrm{sa}}: \sup \{|\omega(d)| \mid \omega \in T(D)\}=\inf \left\{\|d-x\| \mid x \in D_{0}\right\}$, cf. [5] and [1]. Let $\phi:$ Aff $T\left(A / I_{\varphi_{T}}\right) \rightarrow$ Aff $T(B)$ be defined by

$$
\phi(\widehat{\pi(a)})=\varphi_{T_{*}}(\widehat{a}) \quad \forall a \in A_{\mathrm{sa}} .
$$


By Proposition 3.7 of [5], $\pi\left(A_{0}\right)=\left(A / I_{\varphi_{T}}\right)_{0}$. Therefore if $\widehat{\pi(a)}=0$, there exist $b \in A_{0}$ and $x=x^{*} \in I_{\varphi_{T}}$ such that $a=b+x$. Hence $\varphi_{T_{*}}(\widehat{a})=\varphi_{T_{*}}(\widehat{b+x})=$ $\varphi_{T *}(\widehat{x})=0$, by Cauchy-Schwartz. So $\phi$ is well-defined, and clearly $\phi(1)=1$. If $0=\phi\left(\pi\left(\widehat{a^{*} a}\right)\right)=\varphi_{T_{*}}\left(\widehat{a^{*} a}\right)$, then $a^{*} a \in I_{\varphi_{T}}$, so $\phi$ is faithful. $\phi$ is scale preserving, when $\varphi_{T}$ is, because $\widehat{\pi}\left(\Sigma^{t}(A)\right)=\Sigma^{t}\left(A / I_{\varphi_{T}}\right)$.

In the following we will make extensive use of the fact that the class $\mathscr{C}_{\mathrm{T}}$ is closed when taking quotients, if this is not clear to the reader, he is urged to look at Lemma 3.11.

A compatible tuple $\left(\varphi_{0}, \varphi_{T}, \Psi\right)$ is said to be strongly compatible if there exist a group homomorphism $\Phi: U\left(A / I_{\varphi_{T}}\right) / \overline{D U\left(A / I_{\varphi_{T}}\right)} \rightarrow U(B) / \overline{D U(B)}$ such that

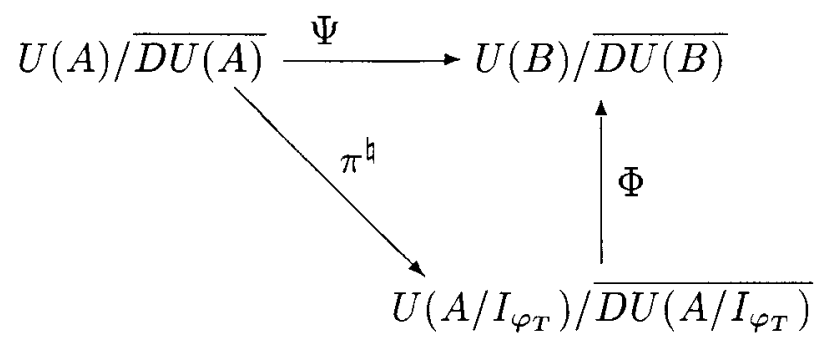

commutes, and a positive homomorphism $\phi_{0}: \mathrm{K}_{0}\left(A / I_{\varphi_{T}}\right) \rightarrow \mathrm{K}_{0}(B)$ such that

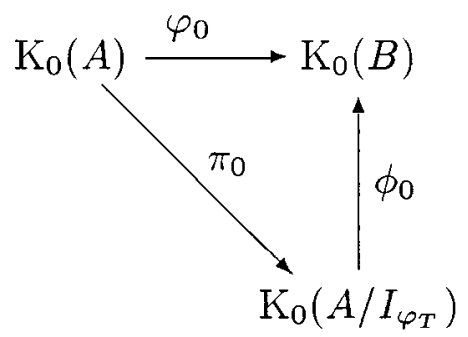

commutes and such that $\phi^{*}$ and $\phi_{0}$ are compatible, i.e.

$$
r_{A / I_{\varphi_{T}}} \circ \phi^{*}(\omega)(x)=r_{B}(\omega)\left(\phi_{0}(x)\right), \quad x \in \mathrm{K}_{0}\left(A / I_{\varphi_{T}}\right), \omega \in T(B),
$$

where $\phi^{*}: T(B) \rightarrow T\left(A / I_{\varphi_{T}}\right)$ is the dual of the map $\phi$ from Lemma 3.1.

As the following lemma shows, a compatible tuple is strongly compatible if and only if, as a morphism $\mathbf{E}(A) \rightarrow \mathbf{E}(B)$, it factorizes through $\mathbf{E}\left(A / I_{\varphi_{T}}\right)$.

Lemma 3.2. Let $A, B \in \mathscr{C}_{\mathrm{T}}$. Assume that there is a positive homomorphism $\varphi_{0}: \mathrm{K}_{0}(A) \rightarrow \mathrm{K}_{0}(B)$, a continuous, affine map $\varphi_{T}: T(B) \rightarrow T(A)$, and a group homomorphism $\Psi: U(A) / \overline{D U(A)} \rightarrow U(B) / \overline{D U(B)}$ such that the tuple $\left(\varphi_{0}, \varphi_{T}, \Psi\right)$ is strongly compatible. Then there exist a (unique) faithful Markov 
operator $\phi:$ Aff $T\left(A / I_{\varphi_{T}}\right) \rightarrow$ Aff $T(B)$, a positive, faithful homomorphism $\phi_{0}: \mathrm{K}_{0}\left(A / I_{\varphi_{T}}\right) \rightarrow \mathrm{K}_{0}(B)$ and a group homomorphism $\Phi: U\left(A / I_{\varphi_{T}}\right) /$ $\overline{D U\left(A / I_{\varphi_{T}}\right)} \rightarrow U(B) / \overline{D U(B)}$ such that $\varphi_{T_{*}}=\phi \circ \widehat{\pi}, \varphi_{0}=\phi_{0} \circ \pi_{0}, \Psi=\Phi \circ \pi^{\natural}$ and such that the tuple $\left(\phi_{0}, \phi^{*}, \Phi\right)$ is (strongly) compatible. Moreover, if $\varphi_{0}$ is order unit preserving, then so is $\phi_{0}$, and if $\varphi_{T}$ is scale preserving, then so is $\phi$.

Proof. The existence of the maps follows from the definition of strongly compatibility and from Lemma 3.1, except that we have to check that $\phi_{0}$ is faithful and that $\lambda_{B} \circ \tilde{\phi}=\Phi \circ \lambda_{A / I_{\varphi_{T}}}$, where $\tilde{\phi}:$ Aff $T\left(A / I_{\varphi_{T}}\right) /$ $\overline{\rho\left(\mathrm{K}_{0}\left(A / I_{\varphi_{T}}\right)\right)} \rightarrow$ Aff $T(B) / \overline{\rho\left(\mathrm{K}_{0}(B)\right)}$ is the well-defined map induced by the compatible maps $\phi$ and $\phi_{0}$. Let $p \in M_{k}\left(A / I_{\varphi_{T}}\right)$ be given and assume that $\phi_{0}([p])=0$. Then for all $\omega \in T(B): 0=r_{B}(\omega)\left(\phi_{0}([p])\right)=\phi(\widehat{p})(\omega)$, thus, since $\phi$ is faithful, $p=0$. Consider the diagram

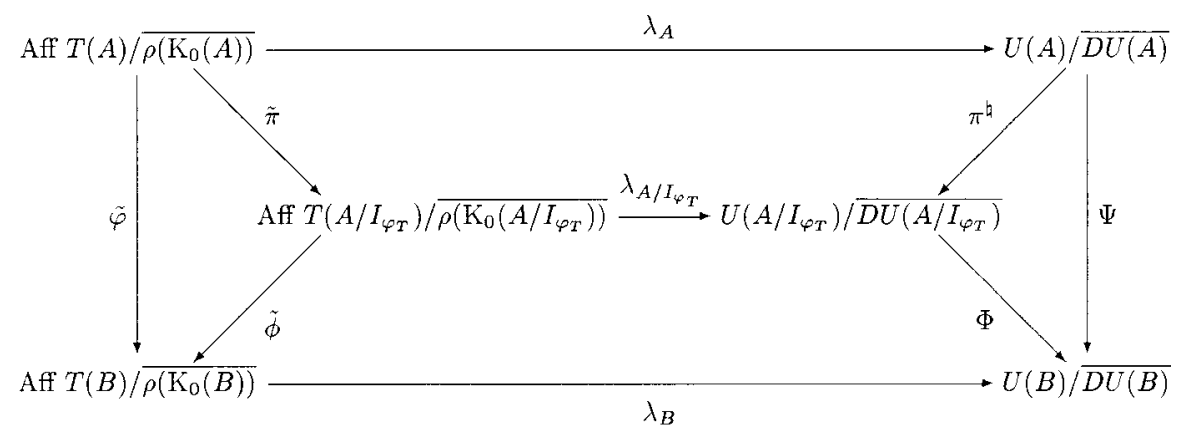

The two triangles, the upper inner square and the outer square commutes. Because $\tilde{\pi}$ is surjective, it follows that so does the lower inner square. When $\varphi_{0}$ is order unit preserving, then $\left[1_{B}\right]=\varphi_{0}\left(\left[1_{A}\right]\right)=\phi_{0}\left(\pi_{0}\left(\left[1_{A}\right]\right)\right)=\phi_{0}\left(\left[1_{A / I_{\varphi_{T}}}\right]\right)$, so $\phi_{0}$ also preserves the unit.

When $A, B \in \mathscr{C}_{\mathrm{\top}}$ and $B$ is simple, then for any unital $*$-homomorphism $\psi: A \rightarrow B$ the dual map $\psi^{*}$ is scale preserving. Moreover, because $B$ is simple, $\operatorname{ker} \psi=I_{\psi^{*}}$, and it follows that the tuple $\left(\psi_{0}, \psi^{*}, \psi^{\natural}\right)$ is strongly compatible. Our goal here is to prove the following existence theorem, which can be considered as the counterpart of Theorem A of [16].

TheOrem 3.3. Let $A, B \in \mathscr{C}_{\top}$ be unital $C^{*}$-algebras with $B$ simple and infinite dimensional. Let $\varphi_{0}: \mathrm{K}_{0}(A) \rightarrow \mathrm{K}_{0}(B)$ be a positive, order unit preserving homomorphism, $\varphi_{T}: T(B) \rightarrow T(A)$ a continuous, scale preserving, affine map and $\Psi: U(A) / \overline{D U(A)} \rightarrow U(B) / \overline{D U(B)}$ a group homomorphism. Then there exists a unital *-homomorphism $\psi: A \rightarrow B$ such that $\psi_{*}=\varphi_{0}$ on $\mathrm{K}_{0}(A)$, $\psi^{*}=\varphi_{T}$ on $T(B)$ and $\psi^{\natural}=\Psi$ on $U(A) / \overline{D U(A)}$ if and only if the tuple $\left(\varphi_{0}, \varphi_{T}, \Psi\right)$ is strongly compatible. Moreover, if $\psi$ exists, then $\operatorname{ker} \psi=I_{\varphi_{T}}$.

As an immediate corollary of Theorem 3.3, we have the following. 
Corollary 3.4. Let $A, B \in \mathscr{C}_{\mathrm{T}}$ be unital $C^{*}$-algebras with $B$ simple and infinite dimensional. Let $\Lambda=\left(\varphi_{0}, \varphi_{T}, \varphi_{1}\right): \mathrm{E}(A) \rightarrow \mathrm{E}(B)$ be a morphism between the Elliott Invariants. Then $\Lambda$ is liftable to a unital *-homomorphism $\psi: A \rightarrow B$ if and only if $\varphi_{T}$ is scale preserving and $\Lambda$ factorizes through $A / I_{\varphi_{T}}$, i.e. there is a morphism $\Gamma: \mathrm{E}\left(A / I_{\varphi_{T}}\right) \rightarrow \mathrm{E}(B)$ such that

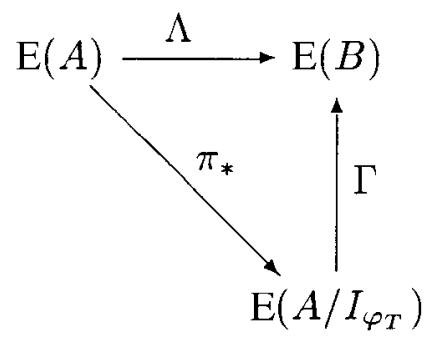

commutes.

REMARK 3.5. Unlike the case considered in Theorem A of [16], in the present situation when given a liftable morphism $\left(\varphi_{0}, \varphi_{T}, \varphi_{1}\right): \mathrm{E}(A) \rightarrow \mathrm{E}(B)$, not every extension of $\left(\varphi_{0}, \varphi_{T}, \varphi_{1}\right)$ to a morphism $\left(\varphi_{0}, \varphi_{T}, \Psi\right): \mathbf{E}(A) \rightarrow \mathbf{E}(B)$ needs to be liftable, cf. Example 3.9.

Before proving Theorem 3.3 let us show that neither the condition of scale preservingness nor the conditions making up the strongly compatibility can be relaxed. In all the examples the domain algebra $A$ will be $C(\mathrm{~T})$ and the map $\left(\varphi_{0}, \varphi_{1}\right): \mathrm{K}_{*}(A) \rightarrow \mathrm{K}_{*}(B)$ will be positive w.r.t. the ordering on $\mathbf{K}_{*}(\cdot)=\mathbf{K}_{0}(\cdot) \oplus \mathbf{K}_{1}(\cdot)$ introduced by Elliott in [8].

Example 3.6. Let $A=C(\mathrm{~T})$ and let $B \in \mathscr{C}_{\mathrm{T}}$ be simple with $\mathrm{K}_{0}(B)=\mathrm{Q}$ as ordered group with order unit $1, \mathrm{~K}_{1}(B)=0$ and Aff $T(B)=\mathrm{R} \oplus \mathrm{R}$ as order unit space with order unit $(1,1)$. Such an algebra exists by Theorem 4.2 of [23]. Now let $\varphi_{0}: \mathrm{K}_{0}(A) \rightarrow \mathrm{K}_{0}(B)$ be the only order unit preserving map and let $\varphi_{1}: \mathrm{K}_{1}(A) \rightarrow \mathrm{K}_{1}(B)$ be the zero map. Choose Borel measures $\mu, \nu$ on $\mathrm{T}$ such that $\operatorname{supp}(\mu) \cup \operatorname{supp}(\nu)=\mathrm{T}$ and such that $\operatorname{supp}(\mu) \cap \operatorname{supp}(\nu)$ is a proper subset of $\mathrm{T}$. Let $\varphi_{T_{*}}$ : Aff $T(A) \simeq C_{\mathrm{R}}(\mathrm{T}) \rightarrow$ Aff $T(B)$ be the map $g \mapsto\left(\int_{\mathrm{T}} g d \mu, \int_{\mathrm{T}} g d \nu\right)$. Set $\Psi_{12}=\Psi_{21}=\Psi_{22}=0$ and $\Psi_{11}=\widetilde{\varphi}$. Then the tuple $\left(\varphi_{0}, \varphi_{T}, \Psi\right)$ is strongly compatible, since $\varphi_{T_{*}}$ is faithful. But $\varphi_{T_{*}}$ is not scale preserving, because by construction there exists an element $f \in \Sigma^{t}(A)$ such that $\varphi_{T_{*}}(f) \notin\{(x, y) \mid x, y>0\} \cup\{(0,0)\}=\Sigma^{t}(B)$.

ExAmple 3.7. [ $\varphi_{0}$ does not factorize] Let $B$ be the CAR-algebra $M_{2 \infty}$ and let $A=C(\mathrm{~T})$. Let $\varphi_{0}: \mathrm{K}_{0}(A) \rightarrow \mathrm{K}_{0}(B)$ be the unique order unit preserving, positive map. Choose three different elements $\lambda_{1}, \lambda_{2}, \lambda_{3} \in \mathrm{T}$ and define $\varphi_{T_{*}}:$ Aff $T(A) \simeq C_{\mathrm{R}}(\mathrm{T}) \rightarrow$ Aff $T(B) \simeq \mathrm{R}$ by $g \mapsto \frac{1}{3} \sum_{i=1}^{3} g\left(\lambda_{i}\right)$. Then $\varphi_{T *}$ is scale preserving and $\left(\varphi_{0}, \varphi_{T}, 0\right)$ is a compatible tuple. $\phi:$ Aff $T\left(A / I_{\varphi_{T}}\right) \simeq$ 
$\mathrm{R}^{3} \rightarrow$ Aff $T(B)$ is the map $\left(x_{1}, x_{2}, x_{3}\right) \mapsto \frac{1}{3} \sum_{i=1}^{3} x_{i}$. Therefore, in particular, $\phi(1,0,0) \in$ Aff $T(B) \backslash \rho\left(\mathrm{K}_{0}(B)\right) \simeq \mathrm{R} \backslash \mathbf{Z}\left[\frac{1}{2}\right]$. But then it follows, that there does not exist any positive, order unit preserving map $\phi_{0}: \mathrm{K}_{0}\left(A / I_{\varphi_{T}}\right) \simeq \mathrm{Z}^{3} \rightarrow \mathrm{K}_{0}(B)$, compatible with $\phi$.

Example 3.8. [ $\varphi_{0}$ factorizes but $\Psi$ does not $-\mathrm{K}_{1}$-obstruction] Choose $B \in \mathscr{C}_{\mathrm{T}}$ simple, infinite dimensional and with only one trace (e.g. $B$ could be an irrational rotation $C^{*}$-algebra) and set $A=C(\mathrm{~T})$. By Theorem 1.3 of [2], $\mathrm{RR}(B)=0$ and from [3] and Theorem 2 of [10] it follows that Aff $T(B)=\overline{\rho\left(\mathrm{K}_{0}(B)\right)}$. Choose a measure $\mu \in M_{1}^{+}(\mathrm{T})$, such that the support of $\mu$ is a proper subset of T homeomorphic to [0,1]. Let $\varphi_{T}: T(B) \rightarrow T(A)$ be the scale preserving map sending the unique trace on $B$ onto integration w.r.t. $\mu$. Let $\varphi_{0}: \mathrm{K}_{0}(A) \rightarrow \mathrm{K}_{0}(B)$ be order unit preserving, and let $\Psi: U(A) / \overline{D U(A)} \rightarrow U(B) / \overline{D U(B)} \simeq \mathrm{K}_{1}(B)$ be the homomorphism with $0=\Psi_{11}=\Psi_{12}=\Psi_{21}$ and where $\Psi_{22}: \mathrm{K}_{1}(A) \rightarrow \mathrm{K}_{1}(B)$ is any non-zero homomorphism. Then the tuple $\left(\varphi_{0}, \varphi_{T}, \Psi\right)$ is compatible and, because $\mathrm{K}_{0}(A) \simeq \mathrm{K}_{0}\left(A / I_{\varphi_{T}}\right)$, we have that $\varphi_{0}$ factorizes through $\mathrm{K}_{0}\left(A / I_{\varphi_{T}}\right)$ in a way compatible with $\varphi_{T}$. But, because $\Psi_{22}$ is non-zero and $\mathrm{K}_{1}\left(A / I_{\varphi_{T}}\right)=0, \Psi$ cannot factorize through $U\left(A / I_{\varphi_{T}}\right) / \overline{D U\left(A / I_{\varphi_{T}}\right)}$.

Example 3.9. [ $\varphi_{0}$ factorizes but $\Psi$ does not - cross map obstruction] Let $X$ be a compact metrizable space, which is not the one-point set. Let $B \in \mathscr{C}_{\mathrm{T}}$ be simple with $\mathrm{K}_{0}(B)=\mathrm{Q}$ as a partially ordered dimension group with order unit 1 and with Aff $T(B)=C_{\mathrm{R}}(X)$ as order unit space with unit the constant function 1. Such an algebra exists by Theorem 4.2 of [23]. Set $A=C(\mathrm{~T})$. Let $\varphi_{0}: \mathrm{K}_{0}(A) \rightarrow \mathrm{K}_{0}(B)$ be order unit preserving and let $\varphi_{T_{*}}: C_{\mathrm{R}}(\mathrm{T}) \simeq$ Aff $T(A) \rightarrow$ Aff $T(B)$ be the scale preserving map $g \mapsto g(1)$. Then it follows that $\varphi_{T_{*}}(\operatorname{Aff} T(A)) \subseteq \overline{\rho\left(\mathrm{K}_{0}(B)\right)}$, and thus that $\widetilde{\varphi}:$ Aff $T(A) / \overline{\rho\left(\mathrm{K}_{0}(A)\right)} \rightarrow$ Aff $T(B) / \overline{\rho\left(\mathrm{K}_{0}(B)\right)}$ is the zero map. Since $\operatorname{RR}(B) \neq 0$, it follows from Theorem 1.3 of [2] that we can choose a non-zero element $y \in$ Aff $T(B) / \overline{\rho\left(\mathrm{K}_{0}(B)\right)} . \quad$ Let $\quad \Psi:\left(C_{\mathrm{R}}(\mathrm{T}) / \mathrm{Z}\right) \oplus \mathrm{Z} \simeq U(A) / \overline{D U(A)} \rightarrow U(B) /$ $\overline{D U(B)}$ be the homomorphism $\left(z_{1}, z_{2}\right) \mapsto\left(z_{2} \cdot y, 0\right)$. Then the tuple $\left(\varphi_{0}, \varphi_{T}, \Psi\right)$ is compatible. Moreover, $\varphi_{0}$ factorizes in a way compatible with $\varphi_{T}$ and so does $\Psi_{22}$ at the level of $\mathrm{K}_{1}$. But, because $\mathrm{K}_{1}\left(A / I_{\varphi_{T}}\right)=0, \tilde{\phi}=0$ and $\Psi_{12} \neq 0$, $\Psi$ cannot factorize in the desired way.

Finally it should be emphasized that Theorem 3.3 cannot be extended to the case of $B$ being finite dimensional, as can be seen from the following example.

Example 3.10. Let $A=C(\mathrm{~T})$ and $B=M_{n}$. Let $\varphi_{0}: \mathrm{K}_{0}(A) \rightarrow \mathrm{K}_{0}(B)$ be the map defined by $[1 \mapsto n]$ (the only possible order unit preserving, positive map). Let $\varphi_{T_{*}}:$ Aff $T(A) \rightarrow$ Aff $T(B)$ be the map $\left[f \mapsto \int_{\mathrm{T}} f d \mu\right]$, where 
$\mu \in M_{1}^{+}(\mathbf{T})$ denote the Lebesgue Measure. Then $\varphi_{T *}$ is scale preserving and faithful. Choose a homomorphism $\delta \in \operatorname{Hom}(\mathrm{Z}, \mathrm{T})$ and define $\Psi: U(A) /$ $\overline{D U(A)} \rightarrow U(B) / \overline{D U(B)}$ as the homomorphism $\Psi_{11}=\widetilde{\varphi}, \Psi_{22}=0=\Psi_{21}$ and $\Psi_{12}=\delta$. Then the tuple $\left(\varphi_{0}, \varphi_{T}, \Psi\right)$ is strongly compatible. But, because $\varphi_{T *}$ is faithful, there is no chance of realizing it from a $*$-homomorphism $A \rightarrow B$.

A unital $C^{*}$-algebra, which is isomorphic to an algebra of the form

$$
\left(\oplus_{j=1}^{K} C\left(X_{j}\right) \otimes M_{n_{j}}\right) \oplus G,
$$

where $X_{j} \in\{[0,1], T\}, j=1, \cdots, K$, and $G$ is finite dimensional, will be called a circle-quotient. Given a $*$-homomorphism $\varphi: A \rightarrow B$ between two circlequotients, then we set mult $\varphi=\min \left\{a_{i j} \mid i, j\right\}$, where $\left(a_{i j}\right)_{i j}$ is an integer matrix representing the map $\varphi_{0}: \mathrm{K}_{0}(A) \rightarrow \mathrm{K}_{0}(B)$.

In order to prove Theorem 3.3 we will need the following two lemmas

LemMa 3.11 .

(1) If $A \in \mathscr{C}_{\mathrm{T}}$, then $A$ can be realized as the inductive limit of a sequence of circle-quotients with injective connective $*$-homomorphisms.

(2) If $A \in \mathscr{C}_{\mathrm{T}}$, then so is any quotient $A / I$ of $A$.

Proof. Given $A \in \mathscr{C}_{\mathrm{T}}$ and $I \subseteq A$ an ideal (possible the zero-ideal). Let $\left\{A_{n}, \mu_{n}\right\}$ be the generating sequence of $A$. Setting $I_{n}=\mu_{\infty, n}^{-1}(I), n \in \mathrm{N}$, then $A / I=\lim \left\{A_{n} / I_{n}, \eta_{n}\right\}$, where the connecting $*$-homomorphisms are all injective. Using Lemma 1.3 of [16], it is straightforward to prove that for any finite subset $F \subseteq A / I$ and $\epsilon>0$, there exists a unital $C^{*}$-subalgebra $B \subseteq A / I$ such that $B$ is a circle-quotient and $F \subseteq_{\epsilon} B$ (cf. the proof of Theorem 1.1 of [16]). Now, from Lemma 1.4 of [16], which easily is seen also to be valid with our definition of a circle-quotient, it follows that $A / I$ is the inductive limit of a sequence of circle-quotients with injective connective $*$-homomorphisms. If $I=0$, this yields (1). (2) follows from the fact that any inductive limit of a sequence of circle-quotients also can be realized as an inductive limit of a sequence of circle algebras.

Lemma 3.12. Let $A=\left(\oplus_{j=1}^{K} C\left(X_{j}\right) \otimes M_{n_{j}}\right) \oplus G$ be a circle-quotient and $B=\oplus_{i=1}^{L} C(\mathrm{~T}) \otimes M_{m_{i}}$. Let $F \subset \operatorname{Aff} T(A)$ be a finite subset. Let $M:$ Aff $T(A) \rightarrow$ Aff $T(B)$ be a Markov operator and $h: \mathrm{K}_{0}(A) \rightarrow \mathrm{K}_{0}(B) a$ group homomorphism such that $M \circ \rho=\rho \circ h: \mathrm{K}_{0}(A) \rightarrow \operatorname{Aff} T(B)$. Given a $\delta>0$, then there exists an integer $T \in \mathrm{N}$ such that for any finite dimension $C^{*}$ algebra $H=M_{l_{1}} \oplus M_{l_{2}} \oplus \cdots \oplus M_{l_{R}}$ with $\min _{j} l_{j} \geq T$, there is a unital *homomorphism $\psi: A \rightarrow B \otimes H$ such that 
(1) $\psi_{*}=\otimes_{1 *} \circ h$ on $\mathrm{K}_{0}(A)$,

(2) $\psi_{*}=0$ on $\mathrm{K}_{1}(A)$, and

(3) $\left\|\widehat{\psi}(f)-\widehat{\otimes_{1}} \circ M(f)\right\|<\delta \forall f \in F$,

where $\otimes_{1}: B \rightarrow B \otimes H$ denotes the $*$-homomorphism $\otimes_{1}(b)=b \otimes 1_{H}$.

Proof. This can be proved by mimicking the proofs of Lemma 4.2 and Corollary 4.3 of [16], using Theorem 2.1 of [21] (the Krein-Milman Theorem for Markov Operators) on Markov operators : $C([0,1]) \rightarrow C(\mathrm{~T})$, and Lemma 4.1 of [16].

Thanks to Lemma 3.2 and Lemma 3.11 it is possible to prove Theorem 3.3 using exactly the same method as in the proof of Theorem A of [16].

Proof of Theorem 3.3. From Lemma 3.2 and Lemma 3.11 it follows that we can assume that $\varphi_{T *}$ and $\varphi_{0}$ both are faithful, and that $A$ is the inductive limit of a sequence of circle quotients with injective connecting $*$-homomorphisms. Let $\left\{A_{n}, \mu_{n}\right\}$ be a generating sequence of $A$. By assumption $B$ is infinite dimensional, and therefore, since it is simple and in $\mathscr{C}_{\mathrm{T}}$, approximately divisible by Theorem 2 of [10]. Let $\left\{B_{n}, \rho_{n}\right\}$ be a generating sequence of $B$.

Proceeding as in the proof of Theorem A of [16], we start by proving the following two assertions.

Assertion 1. For every $n \in \mathrm{N}$, any finite subset $F \subset \operatorname{Aff} T\left(A_{n}\right)$ and any $\epsilon>0$ there is an $m \in \mathrm{N}$ and

(1) a Markov operator $M:$ Aff $T\left(A_{n}\right) \rightarrow$ Aff $T\left(B_{m}\right)$ such that $\left\|\widehat{\rho_{\infty, m}} \circ M(f)-\varphi_{T *} \circ \widehat{\mu_{\infty, n}}(f)\right\|<\epsilon, \forall f \in F$, and

(2) a group homomorphism $h: \mathrm{K}_{0}\left(A_{n}\right) \rightarrow \mathrm{K}_{0}\left(B_{m}\right)$ such that $\rho_{\infty, m_{*}} \circ$ $h=\varphi_{0} \circ \mu_{\infty, n_{*}}$, such that $h$ and $M$ are compatible in the sense that $M \circ \rho(x)=\rho \circ h(x) \forall x \in \mathrm{K}_{0}\left(A_{n}\right)$.

Proof. The proof of Assertion 1 of [16] applies when the simplicity of $A$ used there, is replaced by the fact that because $B$ is simple, then for any nonzero projection $p \in B$ there exists a $\delta_{0}>0$ such that $\widehat{p}>\delta_{0}$.

Assertion 2. For any $n \in \mathrm{N}$, any finite subsets $F_{1} \subset \operatorname{Aff} T\left(A_{n}\right)$ and $F_{2} \subset U\left(A_{n}\right) / \overline{D U\left(A_{n}\right)}$ and any $\epsilon>0$. There is a $k \in \mathrm{N}$ and a unital *-homomorphism $\psi: A_{n} \rightarrow B_{k}$ such that

(1) $\rho_{\infty, k_{*}} \circ \psi_{*}=\varphi_{0} \circ \mu_{\infty, n_{*}}$ on $\mathrm{K}_{0}\left(A_{n}\right)$,

(2) $\left\|\widehat{\rho_{\infty, k}} \circ \widehat{\psi}(f)-\varphi_{T_{*}} \circ \widehat{\mu_{\infty, n}}(f)\right\|<\epsilon \forall f \in F_{1}$, and

(3) $D_{B}\left(\rho_{\infty, k}^{\natural} \circ \psi^{\natural}(y), \Psi \circ \mu_{\infty, n}^{\natural}(y)\right)<\epsilon \forall y \in F_{2}$.

Proof. Let $\{\cdot\}$ denote the one-point set. Then $A_{n}=\oplus_{d=1}^{K} C\left(X_{d}\right) \otimes M_{n_{d}}$, where $X_{d} \in\{\mathrm{T},[0,1],\{\cdot\}\}, d=1, \cdots, K$. Let $\mathscr{J} \subseteq\{1, \cdots, K\}$ be the subset 
consisting of all the $d$ 's for which $X_{d}=\mathrm{T}$. Set $\mathscr{I}=\{1, \cdots, K\} \backslash \mathscr{J}$. In the following we will only consider the case, where neither $\mathscr{I}$ nor $\mathscr{J}$ is the empty set. Having done that, it should be clear to the reader how to reduce to the other (easier) cases. Let $z \in C(\mathrm{~T}) \otimes M_{m}$ be the unitary $\operatorname{Diag}(\mathrm{id}, 1, \cdots, 1)$, and let $z^{d} \in U\left(A_{n}\right), d \in \mathscr{J}$ denote the unitaries $(1, \cdots, 1, z, 1, \cdots, 1)$, where $z$ is placed in the $d^{\prime}$ th summand of $A_{n}$. Then, by Lemma 3.1 of [16], every element $x \in U\left(A_{n}\right) / \overline{D U\left(A_{n}\right)}$ has a unique representation

$$
x=\lambda_{A_{n}}\left(a^{x}\right) \prod_{d \in \mathscr{J}} Q\left(z^{d}\right)^{k_{d}^{x}},
$$

where $a^{x} \in \operatorname{Aff} T\left(A_{n}\right) / \overline{\rho\left(\mathrm{K}_{0}\left(A_{n}\right)\right)}$ and $k_{d}^{x} \in \mathbf{Z}, d \in \mathscr{J}$. For each $y \in F_{2}$ choose a $b^{y} \in$ Aff $T\left(A_{n}\right)$ such that $q\left(b^{y}\right)=a^{y}$. Set $F_{3}=F_{1} \cup\left\{b^{y} \mid y \in F_{2}\right\}$.

Let $0<\delta<2$ be very small; how small is to be specified below. Using Assertion 1, Lemma 3.12, and the fact that $B$ is approximately divisible, it is possible to find a $k \in \mathrm{N}$ and a unital $*$-homomorphisms

$$
\psi_{2}: A_{n} \rightarrow B_{k},
$$

such that

(4) $\psi_{2 *}=0$ on $\mathrm{K}_{1}\left(A_{n}\right)$,

(5) $\varphi_{0} \circ \mu_{\infty, n_{*}}=\rho_{\infty, k_{*}} \circ \psi_{2_{*}}$ on $\mathrm{K}_{0}\left(A_{n}\right)$, and

(6) $\left\|\widehat{\rho_{\infty, k}} \circ \widehat{\psi_{2}}(f)-\varphi_{T_{*}} \circ \widehat{\mu_{\infty, n}}(f)\right\|<\delta \quad \forall f \in F_{3}$.

(For details see the proof of Assertion 2 of [16].)

Let $\iota_{I}: \oplus_{d \in \mathscr{I}} C\left(X_{d}\right) \otimes M_{n_{d}} \rightarrow A_{n}$ and $\iota_{\mathscr{I}}: \oplus_{d \in \mathscr{I}} C(\mathrm{~T}) \otimes M_{n_{d}} \rightarrow A_{n}$ be the inclusions into the appropriate summands, and let $\pi_{\mathscr{I}}: A_{n} \rightarrow$ $\oplus_{d \in \mathscr{I}} C\left(X_{d}\right) \otimes M_{n_{d}}$ and $\pi_{\mathscr{f}}: A_{n} \rightarrow \oplus_{d \in \mathscr{I}} C(\mathrm{~T}) \otimes M_{n_{d}}$ denote the corresponding projections. Consider the $*$-homomorphism

$$
\psi_{3}=\psi_{2} \circ \iota_{\mathscr{g}}: \pi_{\mathscr{J}}\left(A_{n}\right) \rightarrow B_{k} .
$$

Then, since $\varphi_{0}$ is faithful and $\mu_{\infty, n}$ is injective, (5) implies that $\psi_{3 *}$ is faithful on $\mathrm{K}_{0}\left(\pi_{\mathcal{f}}\left(A_{n}\right)\right)$. Because $B$ is simple, we can assume that (if necessary by increasing $k$ )

$$
\text { mult } \psi_{3} \geq \max \left\{\frac{2\|f\|}{\delta} \mid f \in \widehat{\pi_{\mathscr{f}}}\left(F_{3}\right)\right\}
$$

and that $U\left(B_{k}\right) / \overline{D U\left(B_{k}\right)}$ contains elements $\omega_{d}, d \in \mathscr{J}$ such that

$$
D_{B}\left(\rho_{\infty, k}^{\natural}\left(\omega_{d}\right), \Psi \circ \mu_{\infty, n}^{\natural}\left(Q\left(z^{d}\right)\right)\right)<\delta \quad \forall d \in \mathscr{J} .
$$

From Lemma 3.1 of [20] it follows that for any $D \in \mathscr{C}_{\top}$ we have that $\overline{D U_{0}(D)}=U_{0}(D) \cap \overline{D U(D)}$. Combining this with Proposition 2.4 of [22] one gets that with $B_{k}=\oplus_{i=1}^{L} C(\mathrm{~T}) \otimes M_{m_{i}} ; \overline{D U\left(B_{k}\right)}=\left\{\left(u_{1}, \cdots, u_{L}\right) \in U\left(B_{K}\right) \mid\right.$ 
$\left.\operatorname{det}\left(u_{i}\right)=1, i=1, \cdots, L\right\}$. Set $p_{\mathscr{f}}=\psi_{3}(1)$. Since mult $\psi_{3} \geq 1$, it follows that we can find unitaries $u_{d} \in p_{\mathscr{J}} B_{k} p_{\mathscr{J}}, d \in \mathscr{J}$ such that $\omega_{d}=Q\left(u_{d}+1-p_{\mathscr{J}}\right)$ $\forall d \in \mathscr{J}$. Set $\omega_{d}^{\prime}=Q\left(u_{d}\right)$ in $U\left(p_{\mathscr{J}} B_{k} p_{\mathscr{J}}\right) / \overline{D U\left(p_{\mathscr{J}} B_{k} p_{\mathscr{J}}\right)}$. Let $\psi_{4}$ denote the $*-$ homomorphism:

$$
\operatorname{Ad} p_{\mathscr{J}} \circ \psi_{3}: \pi_{\mathscr{f}}\left(A_{n}\right) \rightarrow p_{\mathscr{J}} B_{k} p_{\mathscr{F}} .
$$

Then mult $\psi_{4}=$ mult $\psi_{3}$. By Lemma 3.3 of [16] and by (4), we can construct a unital $*$-homomorphism $\psi_{5}: \pi_{\mathscr{f}}\left(A_{n}\right) \rightarrow p_{\mathscr{f}} B_{k} p_{\mathscr{f}}$ satisfying that $\psi_{5_{*}}=\psi_{4_{*}}$ on $\mathrm{K}_{0}\left(\pi_{\mathscr{L}}\left(A_{n}\right)\right), \quad \psi_{5}^{\natural}\left(Q\left(\pi_{\mathscr{J}}\left(z^{d}\right)\right)\right)=\omega_{d}^{\prime} \forall d \in \mathscr{J} \quad$ and further that $\left\|\widehat{\psi_{5}}(f)-\widehat{\psi_{4}}(f)\right\|<\delta+2\|f\|\left(\text { mult } \psi_{3}\right)^{-1}<2 \delta \forall f \in \widehat{\pi_{\mathcal{f}}}\left(F_{3}\right)$. Now define a unital $*$-homomorphisms $\psi: A_{n} \rightarrow B_{k}$ by

$$
\psi(a)=\psi_{2} \circ \iota_{\mathscr{I}} \circ \pi_{\mathscr{I}}(a)+\psi_{5} \circ \pi_{\mathscr{J}}(a) \quad \forall a \in A_{n} .
$$

Note that by construction $\psi^{\natural}\left(Q\left(z^{d}\right)\right)=\omega_{d} \forall d \in \mathscr{J}$. By (5) one immediately gets that $\psi$ satisfies (1) from above. Furthermore, for all $f \in F_{3} \supseteq F_{1}$

$$
\begin{aligned}
& \left\|\widehat{\rho_{\infty, k}} \circ \widehat{\psi}(f)-\varphi_{T_{*}} \circ \widehat{\mu_{\infty, n}}(f)\right\| \leq \\
& \left\|\widehat{\rho_{\infty, k}} \circ \widehat{\psi}(f)-\widehat{\rho_{\infty, k}} \circ \widehat{\psi_{2}}(f)\right\|+\left\|\widehat{\rho_{\infty, k}} \circ \widehat{\psi_{2}}(f)-\varphi_{T_{*}} \circ \widehat{\mu_{\infty, n}}(f)\right\| \leq \\
& \left\|\widehat{\psi}\left(\left(\widehat{\iota_{\mathscr{I}}} \circ \widehat{\pi_{\mathscr{I}}}+\widehat{\iota_{\mathscr{g}}} \widehat{\pi_{\mathscr{f}}}\right)(f)\right)-\widehat{\psi_{2}}\left(\left(\widehat{\iota_{\mathscr{I}}} \circ \widehat{\pi_{\mathscr{I}}}+\widehat{l_{\mathscr{g}}} \widehat{\pi_{\mathscr{f}}}\right)(f)\right)\right\|+\delta \leq \\
& \left\|\widehat{\psi} \circ \widehat{l_{\mathscr{g}}} \circ \widehat{\pi_{\mathcal{f}}}(f)-\widehat{\psi_{2}} \circ \widehat{l_{\mathscr{f}}} \circ \widehat{\pi_{\mathscr{f}}}(f)\right\|+\delta= \\
& \left\|\widehat{\psi_{5}}\left(\widehat{\pi_{\mathscr{f}}}(f)\right)-\widehat{\psi_{4}}\left(\widehat{\pi_{\mathscr{f}}}(f)\right)\right\|+\delta \leq \\
& 2 \delta+\delta \leq 3 \delta \text {. }
\end{aligned}
$$

Whence if $\delta$ is chosen such that $3 \delta<\epsilon$, then $\psi$ also satisfies (2). Now what is left, is to verify that (3) can be achieved by choosing $\delta$ small enough; For $y \in F_{2}$ :

$\rho_{\infty, k}^{\natural} \circ \psi^{\natural}(y)=\rho_{\infty, k}^{\natural} \circ \psi^{\natural}\left(\lambda_{A_{n}}\left(a^{y}\right) \prod_{d \in \mathscr{J}} Q\left(z^{d}\right)^{k_{d}^{y}}\right)=\lambda_{B}\left(\widetilde{\rho_{\infty, k}} \circ \widetilde{\psi}\left(a^{y}\right)\right) \prod_{d \in \mathscr{J}} \rho_{\infty, k}^{\natural}\left(\omega_{d}\right)^{k_{d}^{y}}$

and

$$
\begin{aligned}
\Psi \circ \mu_{\infty, n}^{\natural}(y) & =\Psi \circ \mu_{\infty, n}^{\natural} \circ\left(\lambda_{A_{n}}\left(a^{y}\right) \prod_{d \in \mathscr{F}} Q\left(z^{d}\right)^{k}\right) \\
& =\lambda_{B}\left(\widetilde{\varphi} \circ \widetilde{\mu_{\infty, n}}\left(a^{y}\right)\right) \prod_{d \in \mathscr{F}}\left(\Psi \circ \mu_{\infty, n}^{\natural}\left(Q\left(z^{d}\right)\right)\right)^{k y_{d}} .
\end{aligned}
$$

The distance in $U(B) / \overline{D U(B)}$ from the element $\rho_{\infty, k}^{\natural} \circ \psi^{\natural}(y)$ to the element

$$
\lambda_{B}\left(\widetilde{\rho_{\infty, k}} \circ \widetilde{\psi}\left(a^{y}\right)\right) \prod_{d \in \mathscr{J}}\left(\Psi \circ \mu_{\infty, n}^{\natural} \circ\left(Q\left(z^{d}\right)\right)\right)^{k_{d}^{y}}
$$

is by (7) less than $\delta \cdot \sum_{d \in \mathscr{Y}}\left|k_{d}^{y}\right|$. Further, the distance between this element 
and the element $\Psi \circ \mu_{\infty, n}^{\natural}(y)$ is equal to the distance

$$
D_{B}\left(\lambda_{B}\left(\widetilde{\rho_{\infty, k}} \circ \widetilde{\psi}\left(a^{y}\right)\right), \lambda_{B}\left(\widetilde{\varphi} \circ \widetilde{\mu_{\infty, n}}\left(a^{y}\right)\right)\right),
$$

which by Lemma 3.1 of [16] is equal to

$$
d_{B}\left(\widetilde{\rho_{\infty, k}} \circ \widetilde{\psi}\left(a^{y}\right), \widetilde{\varphi} \circ \widetilde{\mu_{\infty, n}}\left(a^{y}\right)\right),
$$

which again is smaller than $\left|e^{2 \pi i 3 \delta}-1\right|$ provided that $3 \delta<\frac{1}{2}$. It follows that if $\delta>0$ is chosen such that $3 \delta<\frac{1}{2}$ and

$$
\delta \max \left\{\sum_{d \in \mathscr{J}}\left|k_{d}^{y}\right| \mid y \in F_{2}\right\}+\left|e^{2 \pi i 3 \delta}-1\right|<\epsilon,
$$

then

$$
D_{B}\left(\rho_{\infty, k}^{\natural} \circ \psi^{\natural}(y), \Psi \circ \mu_{\infty, n}^{\natural}(y)\right)<\epsilon \quad \forall y \in F_{2},
$$

and the proof of the assertion is completed.

Construction of the *-homomorphism. We are now ready to construct a unital $*$-homomorphism $\psi: A \rightarrow B$ with the desired properties. As usual this is done by establishing an approximate intertwining in the sense of Elliott (cf. Theorem 2.2 of [8]).

Choose finite subsets $F_{n} \subset$ Aff $T\left(A_{n}\right)$ and $G_{n} \subset U\left(A_{n}\right) / \overline{D U\left(A_{n}\right)}$, such that $\widehat{\mu_{n}}\left(F_{n}\right) \subset F_{n+1}, \mu_{n}^{\natural}\left(G_{n}\right) \subset G_{n+1}$ and $\bigcup_{n} \widehat{\mu_{\infty, n}}\left(F_{n}\right), \bigcup_{n} \mu_{\infty, n}^{\natural}\left(G_{n}\right)$ are dense in Aff $T(A)$ and $U(A) / \overline{D U(A)}$, respectively. Choose a sequence $\left(\delta_{n}\right)_{n}, \delta_{n}>0$, such that $\|\lambda(a)-\eta(a)\|<2^{-n} \forall a \in \bigcup_{k=1}^{n-1} \mu_{n, k}\left(\operatorname{cg}\left(A_{k}\right)\right) \cup \operatorname{cg}\left(A_{n}\right)$, whenever $\eta, \lambda: A_{n} \rightarrow B$ are unital $*$-homomorphisms satisfying that $\|\lambda(a)-\eta(a)\|<$ $\delta_{n} \forall a \in \operatorname{cg}\left(A_{n}\right)$.

We will construct sequences $m_{1}<m_{2}<m_{3}<\cdots$ in $\mathrm{N}$ and unital $*$-homomorphisms $\psi_{k}: A_{k} \rightarrow B_{m_{k}}$ such that

$$
\begin{gathered}
\left\|\rho_{m_{k+1}, m_{k}} \circ \psi_{k}(a)-\psi_{k+1} \circ \mu_{k+1, k}(a)\right\|<\delta_{k}, \quad a \in c g\left(A_{k}\right), \\
\left\|\widehat{\rho_{\infty, m_{k}}} \circ \widehat{\psi_{k}}(f)-\varphi_{T_{*}} \circ \widehat{\mu_{\infty, k}}(f)\right\|<2^{-k}, f \in F_{k}, \\
D_{B}\left(\rho_{\infty, m_{k}}^{\natural} \circ \psi_{k}^{\natural}(y), \Psi \circ \mu_{\infty, k}^{\natural}(y)\right)<2^{-k}, y \in G_{k},
\end{gathered}
$$

and

$$
\rho_{\infty, m_{k *}} \circ \psi_{k_{*}}=\varphi_{0} \circ \mu_{\infty, k_{*}} \text { on } \mathrm{K}_{0}\left(A_{k}\right) .
$$

Having done this, it is standard to check, cf. Theorem 2.2 of [8], that the unital $*$-homomorphism $\psi: A \rightarrow B$, defined by 


$$
\psi \circ \mu_{\infty, n}(a)=\lim _{k \rightarrow \infty} \rho_{\infty, m_{k}} \circ \psi_{k} \circ \mu_{k, n}(a) \quad \forall a \in A_{n}, n \in \mathrm{N},
$$

is well-defined, and has the desired properties. The sequences are constructed by induction in the very same manner as in the proof of Theorem A of [16]namely using Assertion 2 together with the uniqueness theorems for *homomorphisms between the various building blocks.

First we need to introduce some notation; Given $n \in \mathrm{N}$, $A_{n}=\oplus_{d=1}^{K} C\left(X_{d}\right) \otimes M_{n_{d}}, \quad$ where $\quad X_{d} \in\{\mathrm{T},[0,1],\{\cdot\}\}, d=1, \cdots, K$. Let $\mathscr{I}, \mathscr{J} \subseteq\{1, \cdots, K\}$ be defined as in the proof of Assertion 2, and let $\mathscr{H} \subseteq \mathscr{I}$ be the subset consisting of all the $d$ 's for which $X_{d}=\{\cdot\}$. Again we will only consider the case, where $\mathscr{I}, \mathscr{J}$ and $\mathscr{H}$ are all non-empty subsets. Let $\pi_{\mathscr{J}}, \pi_{s}$, and $\pi_{f}$ denote the projection of $A_{n}$ onto its component of finite direct sum of circle algebras, the projection of $A_{n}$ onto its component of finite direct sums of interval algebras, and the projection of $A_{n}$ onto its finite dimensional component, respectively. Moreover let $\iota_{\mathscr{f}}, \iota_{s}$ and $\iota_{f}$ denote the corresponding inclusions. Let $b_{i}, i=1, \cdots, s$, be the canonical selfadjoint generators of the center of $\pi_{s}\left(A_{n}\right)$, and set $a_{i}=\iota_{s}\left(b_{i}\right), i=1, \cdots, s$. Finally let $u_{d} \in A_{n}, d \in \mathscr{J}$ denote the partial unitaries which are the canonical generators of the center of $\pi_{\mathscr{J}}\left(A_{n}\right)$, and set $\mathrm{cu}_{\mathscr{J}}\left(A_{n}\right)=\left\{u_{d}+1-u_{d}^{*} u_{d} \mid d \in \mathscr{J}\right\}$.

In order to make the induction work (and in particular to obtain (1)), we have to impose the following conditions:

There are integers $r_{k}, t_{k} \in \mathrm{N}, t_{k}>12$ and numbers $\kappa_{k}>0$ such that $\left(\frac{28}{r_{k}}+\frac{6}{t_{k}}\right) \pi<\delta_{k}$

(6) $\theta\left(\zeta_{j}\left(\psi_{k}\left(a_{i}\right)\right)\right)>2 \kappa_{k} \quad \forall j=1, \cdots, r_{k}, \theta \in T\left(B_{m_{k}}\right), i=1, \cdots, s$,

(7) $\theta\left(\xi_{j}^{r_{k}}\left(\psi_{k}\left(u_{d}\right)\right)\right)>\frac{1}{t_{k}} \forall j=1, \cdots, r_{k}, \theta \in T\left(B_{m_{k}}\right), d \in \mathscr{J}$,

(8) $\theta\left(\xi_{j}^{3 t_{k}}\left(\psi_{k}\left(u_{d}\right)\right)\right)>2 \kappa_{k} \quad \forall j=1, \cdots, 3 t_{k}, \theta \in T\left(B_{m_{k}}\right), d \in \mathscr{J}$,

(9) $D_{B}\left(\rho_{\infty, m_{k}}^{\natural} \circ \psi_{k}^{\natural}(Q(u)), \Psi \circ \mu_{\infty, k^{\natural}}(Q(u))\right)<t_{k}^{-2} \forall u \in \mathrm{cu}_{\mathscr{J}}\left(A_{k}\right)$,

(10) $\left\|\widehat{\rho_{\infty, m_{k}}} \circ \widehat{\psi_{k}}(x)-\varphi_{T_{*}} \circ \widehat{\mu_{\infty, k}}(x)\right\|<\kappa_{k} \forall x \in\left\{h \widehat{h\left(u_{d}\right)}, g \widehat{\left(a_{i}\right)} \mid\right.$ $\left.h \in H_{k}, g \in L_{k}, d \in \mathscr{J}, i=1, \cdots, s\right\}$.

Where the functions $\left\{\zeta_{j}\right\}$ and $\left\{\xi_{j}^{m}\right\}$ are as in the uniqueness theorems, Lemma 2.3 and Theorem 2.4 of [16] respectively. Further $L_{k} \subset C[0,1]$ is a subset meeting the requirements of Lemma 2.3 corresponding to $n=r_{k}$, and $H_{k} \subset C(\mathrm{~T} \cup\{0\},[0,1])$ is a subset meeting the requirements of Theorem 2.4 of [16] corresponding to $m=r_{k}$ and $n=t_{k}$.

Let us start by constructing $r_{1}, t_{1}, \kappa_{1}, m_{1}$ and $\psi_{1}: A_{1} \rightarrow B_{m_{1}}$ : Choose $r_{1} \in \mathrm{N}$ such that $\frac{28 \pi}{r_{1}}<\frac{\delta_{1}}{2}$, next choose $t_{1} \in \mathrm{N}, t_{1}>12$ such that $\frac{6 \pi}{t_{1}}<\frac{\delta_{1}}{2}$ and

$$
\left.\varphi_{T_{*}} \circ \widehat{\mu_{\infty, 1}}\left(\xi_{j}^{r_{1}} \widehat{\left(u_{d}\right.}\right)\right)>\frac{2}{t_{1}} \quad \forall j=1, \cdots, r_{1}, d \in \mathscr{J} .
$$

Then choose $\kappa_{1}>0$ such that 


$$
\varphi_{T_{*}} \circ \widehat{\mu_{\infty, 1}}\left(\zeta_{j} \widehat{\left(a_{i}\right)}\right)>3 \kappa_{1} \quad \forall j=1, \cdots, r_{1}, i=1, \cdots, s
$$

and

$$
\varphi_{T *} \circ \widehat{\mu_{\infty, 1}}\left(\xi_{j}^{3 t t_{1}\left(u_{d}\right)}\right)>3 \kappa_{1} \quad \forall j=1, \cdots, 3 t_{1}, d \in \mathscr{J} .
$$

This is possible because $B$ is simple and $\varphi_{T_{*}} \circ \widehat{\mu_{\infty, 1}}$ is faithful and scale preserving. By Assertion 2, there exist an $m_{1} \in \mathrm{N}$ and a unital $*$-homomorphism $\psi_{1}: A_{1} \rightarrow B_{m_{1}}$ satisfying that $\rho_{\infty, m_{1 *}} \circ \psi_{1 *}=\varphi_{0} \circ \mu_{\infty, 1_{*}}$ on $\mathrm{K}_{0}\left(A_{1}\right)$, $D_{B}\left(\rho_{\infty, m_{1}}{ }^{\natural} \circ \psi_{1}^{\natural}(Q(u)), \Psi \circ \mu_{\infty, 1^{\natural}}(Q(u))\right)<\min \left\{t_{1}^{-2}, 2^{-1}\right\} \quad \forall u \in \operatorname{cu}_{\mathscr{J}}\left(A_{1}\right) \cup G_{1}$, and

$$
\left\|\widehat{\rho_{\infty, m_{1}}} \circ \widehat{\psi_{1}}(x)-\varphi_{T_{*}} \circ \widehat{\mu_{\infty, 1}}(x)\right\|<\min \left\{t_{1}^{-1}, \kappa_{1}, 2^{-1}\right\} \quad \forall x \in J_{1},
$$

with

$$
\begin{aligned}
& J_{1}=F_{1} \cup\left\{\widehat{h\left(u_{d}\right)}, g \widehat{\left(a_{i}\right)} \mid h \in H_{1}, g \in L_{1}, d \in \mathscr{J}, i=1, \cdots, s\right\} \cup \\
& \left\{\xi_{\ell}^{n} \widehat{\left(u_{d}\right)}, \zeta_{j} \widehat{\left(a_{i}\right)} \mid n \in\left\{r_{1}, 3 t_{1}\right\}, \ell=1, \cdots, n, j=1, \cdots, r_{1}, d \in \mathscr{J}, i=1, \cdots, s\right\} .
\end{aligned}
$$

Thus, if necessary by increasing $m_{1}$, we can obtain (2)-(4) and (6)-(10) for $k=1$. Now assume that $m_{1}<m_{2}<\cdots<m_{n}, \quad r_{1}<r_{2}<\cdots<r_{n}$, $t_{1}<t_{2}<\cdots<t_{n}, \quad\left\{\kappa_{i} \mid 1 \leq i \leq n\right\}$ and $\left\{\psi_{i} \mid 1 \leq i \leq n\right\}$ have been constructed fulfilling (2)-(4) and (6)-(10) for all $k \leq n$. Let us then prove, that we can construct $m_{n+1}, r_{n+1}, t_{n+1}, \kappa_{n+1}$ and $\psi_{n+1}: A_{n+1} \rightarrow B_{m_{n+1}}$ fulfilling not only (2)-(4) and (6)-(10) but also (1). Choose $\epsilon>0$ such that (9) and (10) still are valid with $t_{n}^{-2}$ replaced by $t_{n}^{-2}-\epsilon$ and with $\kappa_{n}$ replaced by $\kappa_{n}-\epsilon$, respectively. Let $r_{n+1}, t_{n+1}$ and $\kappa_{n+1}$ be chosen as in the case of $k=1$, using that $\varphi_{T *} \circ \widehat{\mu_{\infty, n+1}}$ is faithful and scale preserving. By Assertion 2 find an $m_{n+1} \in \mathrm{N}$ and a $*$-homomorphisms $\phi: A_{n+1} \rightarrow B_{m_{n+1}}$ satisfying that $\rho_{\infty, m_{n+1 *}} \circ \phi_{*}=\varphi_{0} \circ \mu_{\infty, n+1_{*}}$ on $\mathrm{K}_{0}\left(A_{n+1}\right)$, and

$$
\begin{aligned}
& D_{B}\left(\rho_{\infty, m_{n+1}}^{\natural} \circ \phi^{\natural}(Q(u)), \Psi \circ \mu_{\infty, n+1}^{\natural}(Q(u))\right)<\min \left\{\epsilon, t_{n+1}^{-2}, 2^{-n-1}\right\} \\
& \forall u \in c u\left(A_{n+1}\right) \cup G_{n+1} \cup \mu_{n+1, n}\left(c u_{\mathscr{J}}\left(A_{n}\right)\right),
\end{aligned}
$$

and

$$
\begin{aligned}
& \left\|\widehat{\rho_{\infty, m_{n+1}}} \circ \widehat{\phi}(x)-\varphi_{T_{*}} \circ \widehat{\mu_{\infty, n+1}}(x)\right\|<\min \left\{\epsilon, t_{n+1}^{-1}, \kappa_{n+1}, 2^{-n-1}\right\} \forall x \in \\
& J_{n+1} \cup \widehat{\mu_{n+1}, n}\left(\left\{h \widehat{\left(u_{d}\right)}, g \widehat{\left(a_{i}\right)} \mid h \in H_{n}, g \in L_{n}, d \in \mathscr{J}, i=1, \cdots, s\right\}\right),
\end{aligned}
$$

where $J_{n+1}$ is defined analogously to $J_{1}$. Then, if necessary by increasing $m_{n+1}$, we get that (2)-(4) and (6)-(10) are satisfied for $\psi_{n+1}=\phi$. Moreover by (11)-(12) we can assume that 


$$
\begin{gathered}
D_{B_{m_{n+1}}}\left(\rho_{m_{n+1}, m_{n}}^{\natural} \circ \psi_{n}^{\natural}(Q(u)), \phi^{\natural} \circ \mu_{n+1, n}^{\natural}(Q(u))\right)<t_{n}^{-2} \quad \forall u \in c u_{\mathscr{J}}\left(A_{n}\right), \\
\left\|\widehat{\rho_{m_{n+1}, m_{n}}} \circ \widehat{\psi_{n}}(x)-\widehat{\phi} \circ \widehat{\mu_{n+1}, n}(x)\right\|<\kappa_{n} \quad \forall x \in \\
\left\{h \widehat{\left(u_{d}\right)}, \widehat{g\left(a_{i}\right)} \mid h \in H_{n}, g \in L_{n}, d \in \mathscr{J}, i=1, \cdots, s\right\},
\end{gathered}
$$

and $\rho_{m_{n+1}, m_{n *}} \circ \psi_{n_{*}}=\phi_{*} \circ \mu_{n+1, n_{*}}$ on $\mathrm{K}_{0}\left(A_{n}\right)$. In order to obtain (1) it is enough to prove that there exists a unitary $U \in B_{m_{n+1}}$ such that

$$
\left\|U \rho_{m_{n+1}, m_{n}} \circ \psi_{n}(a) U^{*}-\phi \circ \mu_{n+1, n}(a)\right\|<\delta_{n} \quad \forall a \in \operatorname{cg}\left(A_{n}\right),
$$

because then $\psi_{n+1}=\operatorname{Ad} U^{*} \circ \phi$ will do the job. To simplify the notation set $\phi=\phi \circ \mu_{n+1, n}, \quad \psi=\rho_{m_{n+1}, m_{n}} \circ \psi_{n}, \quad p_{\mathscr{J}}=\iota_{\mathscr{J}} \circ \pi_{\mathscr{J}}(1), \quad p_{s}=\iota_{s} \circ \pi_{s}(1) \quad$ and $p_{f}=\iota_{f} \circ \pi_{f}(1)$. Then, since $\psi$ and $\phi$ have the same action on $\mathrm{K}_{0}$, we can assume that $\psi\left(p_{\mathscr{J}}\right)=\phi\left(p_{\mathscr{J}}\right), \psi\left(p_{s}\right)=\phi\left(p_{s}\right)$ and $\psi\left(p_{f}\right)=\phi\left(p_{f}\right)$. Set $P_{\mathscr{J}}=\psi\left(p_{\mathscr{J}}\right)$, $P_{s}=\psi\left(p_{s}\right)$ and $P_{f}=\psi\left(p_{f}\right)$ and define unital $*$-homomorphisms

$$
\begin{array}{lll}
\psi_{\mathscr{J}}=\operatorname{Ad} P_{\mathscr{J}} \circ \psi \circ \iota_{\mathscr{I}}, \quad \phi_{\mathscr{J}}=\operatorname{Ad} P_{\mathscr{I}} \circ \phi \circ \iota_{\mathscr{J}} & : \pi_{\mathscr{J}}\left(A_{n}\right) \rightarrow P_{\mathscr{I}} B_{m_{n+1}} P_{\mathscr{J}} \\
\psi_{s}=\operatorname{Ad} P_{s} \circ \psi \circ \iota_{s}, \quad \phi_{s}=\operatorname{Ad} P_{s} \circ \phi \circ \iota_{s} & : & \pi_{s}\left(A_{n}\right) \rightarrow P_{s} B_{m_{n+1}} P_{s} \\
\psi_{f}=\operatorname{Ad} P_{f} \circ \psi \circ \iota_{f}, \quad \phi_{f}=\operatorname{Ad} P_{f} \circ \phi \circ \iota_{f} & : & \pi_{f}\left(A_{n}\right) \rightarrow P_{f} B_{m_{n+1}} P_{f}
\end{array}
$$

Since by construction $\psi_{f}$ and $\phi_{f}$ have the same action on $\mathrm{K}_{0}$, there exists a unitary $V_{f} \in P_{f} B_{m_{n+1}} P_{f}$ such that

$$
\text { Ad } V_{f} \circ \psi_{f}=\phi_{f}
$$

By (6), (14) and Lemma 2.3 (and its proof) there exists a unitary $V_{s} \in P_{s} B_{m_{n+1}} P_{s}$ such that

$$
\left\|\operatorname{Ad} V_{s} \circ \psi_{s}(a)-\phi_{s}(a)\right\| \leq \frac{3}{r_{n}}<\delta_{n} \quad \forall a \in \operatorname{cg}\left(\pi_{s}\left(A_{n}\right)\right) .
$$

And finally by (7), (8), (13), (14) and Theorem 2.4 of [16] (and its proof) there exists a unitary $V_{\mathscr{J}} \in P_{\mathscr{J}} B_{m_{n+1}} P_{\mathscr{J}}$ such that

$$
\left\|\operatorname{Ad} V_{\mathscr{J}} \circ \psi_{\mathscr{J}}(a)-\phi_{\mathscr{J}}(a)\right\|<\left(\frac{28}{r_{n}}+\frac{6}{t_{n}}\right) \pi<\delta_{n} \quad \forall a \in \operatorname{cg}\left(\pi_{\mathscr{J}}\left(C_{n}\right)\right) .
$$

Now set $U=V_{\mathscr{J}}+V_{s}+V_{f}$. Then, by (16)-(18), $U \in B_{m_{n+1}}$ is a unitary for which (15) is valid, and we have completed the induction step.

\section{Lifting homomorphisms from K-theory}

In view of the classification of some classes of $C^{*}$-algebras using K-theory alone, cf. [8], [14] and [17], one could ask to what extend a positive (w.r.t. the ordering from [8]) homomorphism $\left(\varphi_{0}, \varphi_{1}\right): \mathrm{K}_{*}(A) \rightarrow \mathrm{K}_{*}(B)$ lifts to a unital 
*-homomorphism $A \rightarrow B$, when $A$ and $B$ are $C^{*}$-algebras from the class $\mathscr{C}_{\mathrm{T}}$. In [8], Elliott proved that for algebras in $\mathscr{C}_{T}$ of real rank zero, then such a lift does always exist. For the larger class of all AD-algebras (inductive limits of sequences of finite direct sums of circle - and/or dimension drops algebras) of real rank zero, Dardalat and Loring in [6] proved that any isomorphism on the level of K-theory lifts, when the algebras are simple (this was later shown by Eilers to be true even when the algebras have at most finitely many ideals, cf. [7]). But Dardalat and Loring also gave an example to the fact that for non-simple $\mathrm{AD}$-algebras of real rank zero a lifting from K-theory alone is not always possible, cf. [6].

For $A, B \in \mathscr{C}_{\mathrm{T}}, A$ being simple and $B$ approximately divisible, the question of whether a positive homomorphism $\left(\varphi_{0}, \varphi_{1}\right): \mathrm{K}_{*}(A) \rightarrow \mathrm{K}_{*}(B)$ can be lifted to a $*$-homomorphism $A \rightarrow B$ is equivalent to the question of whether, for a given positive, order unit preserving homomorphism $\varphi_{0}: \mathrm{K}_{0}(A) \rightarrow \mathrm{K}_{0}(B)$, there exists an affine, continuous map $\varphi_{T}: T(B) \rightarrow T(A)$, compatible with $\varphi_{0}$. Because given two such compatible maps $\varphi_{0}$ and $\varphi_{T}$, then for any homomorphism $\varphi_{1}: \mathrm{K}_{1}(A) \rightarrow \mathrm{K}_{1}(B)$ the tuple $\left(\varphi_{0}, \varphi_{T}, \Psi\right)$ is compatible, if we set $\Psi_{12}=\Psi_{21}=0, \Psi_{11}=\widetilde{\varphi}$ and $\Psi_{22}=\varphi_{1}$. So, by Theorem A of [16], there exists a unital $*$-homomorphism $A \rightarrow B$ realizing $\left(\varphi_{0}, \varphi_{1}\right)$.

Let $D$ be a unital inductive limit of a sequence of finite direct sums of $C^{*}$ algebras of the form $C(X) \otimes M_{n}$, where $X$ is a compact Hausdorff space. Then the trace state space $T(D)$ and the state space of the $\mathrm{K}_{0}$-group $S\left(\mathrm{~K}_{0}(D)\right)$ are both Choquet simplexes by [23]. The pairing map $r_{D}: T(D) \rightarrow S\left(\mathrm{~K}_{0}(D)\right)$ is continuous and surjective, by [4] and [13], since $D$ is exact. Moreover from [23] we know that $r_{D}$ is extreme point preserving, i.e. $r_{D}\left(\partial_{e} T(D)\right)=\partial_{e} S\left(\mathrm{~K}_{0}(D)\right)$.

The following proposition is an immediate consequence of Lazar's Selection Theorem, Theorem 3.1 of [15].

Proposition 4.1. Let $A, B$ be unital inductive limits of sequences of finite direct sums of $C^{*}$-algebras of the form $C(X) \otimes M_{n}$, where $X$ is a compact Hausdorff space. Assume that the pairing map $r_{A}: T(A) \rightarrow S\left(\mathrm{~K}_{0}(A)\right)$ is open. Let $\varphi_{0}: \mathrm{K}_{0}(A) \rightarrow \mathrm{K}_{0}(B)$ be a positive, order unit preserving homomorphism. Then there exists a continuous, affine map $\varphi_{T}: T(B) \rightarrow T(A)$, compatible with $\varphi_{0}$.

Proof. By assumption, $r_{A}: T(A) \rightarrow S\left(\mathrm{~K}_{0}(A)\right)$ is an affine, continuous, surjective, open map between Choquet simplexes. From Theorem 3.1 of [15] it follows that there exists a continuous, affine map $s: S\left(\mathrm{~K}_{0}(A)\right) \rightarrow T(A)$ such that $r_{A} \circ s=\mathrm{id}_{S\left(\mathrm{~K}_{0}(\mathrm{~A})\right)}$. Let $\varphi_{0}^{*}: S\left(\mathrm{~K}_{0}(B)\right) \rightarrow S\left(\mathrm{~K}_{0}(A)\right)$ be the dual map of $\varphi_{0}$. The assignment $\varphi_{T}=s \circ \varphi_{0}{ }^{*} \circ r_{B}$ defines a continuous, affine map $T(B) \rightarrow T(A)$ compatible with $\varphi_{0}$. 
Corollary 4.2. Let $A, B \in \mathscr{C}_{\mathrm{\top}}$ with $A$ simple and $B$ approximately divisible. Let $\varphi_{0}: \mathrm{K}_{0}(A) \rightarrow \mathrm{K}_{0}(B)$ be a positive, order unit preserving homomorphism and let $\varphi_{1}: \mathrm{K}_{1}(A) \rightarrow \mathrm{K}_{1}(B)$ be a group homomorphism. If $r_{A}: T(A) \rightarrow S\left(\mathrm{~K}_{0}(A)\right)$ is open, then there exists a unital *-homomorphism $\psi: A \rightarrow B$ such that $\psi_{*}=\left(\varphi_{0}, \varphi_{1}\right)$ on $\mathrm{K}_{*}(A)$.

Proof. Theorem A of [16] combined with Proposition 4.1.

In general, however, even when dealing with simple $C^{*}$-algebras from the class $\mathscr{C}_{\mathrm{T}}$, not every positive map $\varphi_{0}: \mathrm{K}_{0}(A) \rightarrow \mathrm{K}_{0}(B)$ lifts to a unital $*$ homomorphism $A \rightarrow B$, as can be seen from the following example. In the example, the obstruction to the lifting lies in the fact, that the $\mathrm{K}_{0}$-map cannot be properly paired with any affine, continuous map between the tracial state spaces. This type of obstruction would of course not be present if the algebras were purely infinite or had real rank zero.

ExAmple 4.3. Given a compact Hausdorff space $X$ we let $M_{1}^{+}(X)$ denote the Choquet simplex consisting of all probability measures on $X$. Let $\lambda: M_{1}^{+}([0,1]) \rightarrow M_{1}^{+}(\mathrm{T})$ be the affine, continuous, surjective map $\mu \mapsto \mu \circ h^{-1}$, where $h:[0,1] \rightarrow \mathrm{T}$ is the map $t \mapsto e^{2 \pi i t}$. Let $\delta_{t}, t \in[0,1]$ and $\nu_{z}, z \in \mathrm{T}$ denote the Dirac measures on $[0,1]$ and $\mathrm{T}$ respectively. Then $\partial_{e} M_{1}^{+}([0,1])=\left\{\delta_{t} \mid t \in[0,1]\right\}, \partial_{e} M_{1}^{+}(\mathrm{T})=\left\{\nu_{z} \mid z \in \mathrm{T}\right\}$, and it follows that $\lambda\left(\partial_{e} M_{1}^{+}([0,1])\right)=\partial_{e} M_{1}^{+}(\mathrm{T})$. By Theorem 14.12 of [12], there exists a simple countable dimension group $G \neq \mathrm{Z}$ with order unit $u \in G^{+}$such that the state space $S(G, u) \simeq M_{1}^{+}(\mathrm{T})$. By Theorem 4.2 of [23] there exist simple, unital $C^{*}$ algebras $A, B \in \mathscr{C}_{\mathrm{T}}$ (in fact they can both be chosen as inductive limits of sequences of finite direct sums of interval algebras - Theorem 3.2 of [23]) such that $r_{A}: T(A) \rightarrow S\left(\mathrm{~K}_{0}(A)\right)$ is isomorphic to $\lambda: M_{1}^{+}([0,1]) \rightarrow M_{1}^{+}(\mathrm{T})$ and such that $r_{B}: T(B) \rightarrow S\left(\mathrm{~K}_{0}(B)\right)$ is isomorphic to id $: M_{1}^{+}(\mathrm{T}) \rightarrow M_{1}^{+}(\mathrm{T})$. Now let us assume that there exists a continuous, affine map $\varphi_{T}: T(B) \rightarrow T(A)$ compatible with id $: \mathrm{K}_{0}(A) \rightarrow \mathrm{K}_{0}(B)$. Then the diagram

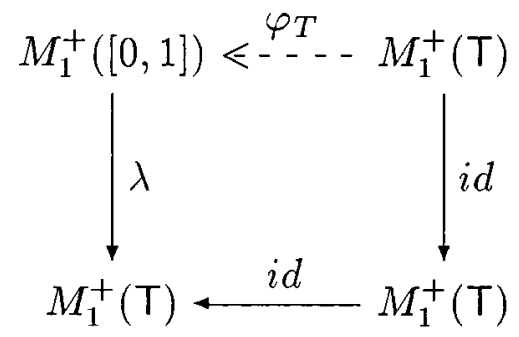

has to be commutative, and it follows that $\varphi_{T}: M_{1}^{+}(\mathrm{T}) \rightarrow M_{1}^{+}([0,1])$ is a continuous, affine section for $\lambda$, i.e. $\lambda \circ \varphi_{T}=\mathrm{id}_{M_{1}^{+}(\mathrm{T})}$. Let $\left.t \in\right] 0,1[$, then $\lambda^{-1}\left(\nu_{e^{2 \pi i t}}\right)$ is a closed face in $M_{1}^{+}([0,1])$ and therefore the closed convex hull 
of its extreme points $\partial_{e} \lambda^{-1}\left(\nu_{e^{2 \pi i t}}\right)=\lambda^{-1}\left(\nu_{e^{2 \pi i t}}\right) \cap \partial_{e} M_{1}^{+}([0,1])=\left\{\delta_{t}\right\}$. Whence it follows that $\left.\varphi_{T}\left(\nu_{e^{2 \pi i t}}\right)=\delta_{t} \forall t \in\right] 0,1\left[\right.$. But then by continuity $\varphi_{T}$ has to map $\nu_{1}$ to both $\delta_{0}$ and $\delta_{1}$, a contradiction. Hence id : $\mathrm{K}_{0}(A) \rightarrow \mathrm{K}_{0}(B)$ cannot be lifted to a unital $*$-homomorphism $A \rightarrow B$.

\section{Unitary Elements}

Theorem 2.1 and Theorem 3.3 in hand, we are able to give sufficient and necessary conditions for two unitaries in a simple $C^{*}$-algebra from $\mathscr{C}_{T}$ to be approximate unitary equivalent. When the algebra in question is of real rank zero, these conditions reduces to the conditions given by Elliott in Theorem 3 , (iii) of [11].

Theorem 5.1. Let $B \in \mathscr{C}_{\mathrm{T}}$ be simple and infinite dimensional. Given unitaries $U, V \in B$, then $U$ and $V$ are approximate unitary equivalent in $B$ if and only if

(1) $\theta(f(U))=\theta(f(V))$ for all $\theta \in T(B)$ and $f \in C(\mathbf{T}, \mathbf{R})$.

(2) $Q(U)=Q(V)$, i.e. the unitaries have the same class in $U(B) / \overline{D U(B)}$.

Furthermore if $\mathrm{R} R(B)=0$, then condition (2) reduces to the condition that the unitaries should have the same class in $\mathrm{K}_{1}(B)$. Conversely if $\mathrm{R} \mathrm{R}(B) \neq 0$ then for any unitary $U \in B$ with $s p(U)=\mathrm{T}$, there exists a unitary $V \in B$ such that $U$ and $V$ satisfy (1) and have the same class in $\mathrm{K}_{1}(B)$, although $U$ and $V$ are not approximate unitary equivalent.

Proof. The necessity of the conditions is obvious. Given two unitaries $U, V \in B$, we define unital $*$-homomorphisms $\varphi_{U}, \varphi_{V}: C(\mathrm{~T}) \rightarrow B$ by the assignment

$$
\varphi_{U}(f)=f(U) \text { and } \varphi_{V}(f)=f(V) \text { for all } f \in C(\mathbf{T}) .
$$

Now if the pair $U, V$ satisfies condition (1) and (2), then it is easy to see that $\varphi_{U}$ and $\varphi_{V}$ satisfy the conditions in Theorem 2.1. Whence it follows that $\varphi_{U}$ and $\varphi_{V}$ are approximate unitary equivalent, and so, in particular, are $U$ and $V$.

By Theorem 2 of [10], $B$ is approximately divisible, since simple and infinite dimensional. Therefore, by [3], $\operatorname{RR}(B)=0$ if and only if $\overline{\rho\left(\mathrm{K}_{0}(B)\right)}=$ Aff $T(B)$. So when $\operatorname{RR}(B)=0$, the group $\operatorname{Hom}(\mathrm{Z}, \operatorname{Aff} T(B) /$ $\left.\overline{\rho\left(\mathrm{K}_{0}(B)\right)}\right)$ is trivial. Thus given any $*$-homomorphism $\psi: C(\mathrm{~T}) \rightarrow B$, the homomorphism $\psi^{\natural}$ is uniquely determined by the maps $\widetilde{\psi}$ and $\psi_{1}$. This, applied to the $*$-homomorphisms $\varphi_{U}, \varphi_{V}$ together with Theorem 2.1, yields that condition (2) reduces in the described way. Conversely, assume that Aff $T(B) / \overline{\rho\left(\mathrm{K}_{0}(B)\right)}$ contains more than the trivial element. Given a unitary $U \in B$ with $\operatorname{sp}(U)=\mathrm{T}$, the $*$-homomorphism $\varphi_{U}: C(\mathrm{~T}) \rightarrow B$ is injective, so 
in particular the scale preserving map $\widehat{\varphi_{U}}: C_{\mathrm{R}}(\mathrm{T}) \rightarrow \operatorname{Aff} T(B)$ is faithful. Choose a non-zero element $x \in$ Aff $T(B) / \overline{\rho\left(\mathrm{K}_{0}(B)\right)}$ and let $\delta: Z \rightarrow$ Aff $T(B) / \overline{\rho\left(\mathrm{K}_{0}(B)\right)}$ be the homomorphism defined by $[1 \mapsto x]$. Now let $\Psi: U(C(\mathrm{~T})) / \overline{D U(C(\mathrm{~T})}) \rightarrow U(B) / \overline{D U(B)}$ be the homomorphism defined by the decomposition $\Psi_{11}=\widetilde{\varphi_{U}}, \Psi_{22}=\varphi_{U 1}, \Psi_{12}=\varphi_{U 12}^{\natural}+\delta$ and $\Psi_{21}=0$. Then the tuple $\left(\varphi_{U_{0}}, \varphi_{U}{ }^{*}, \Psi\right)$ is strongly compatible. Hence, by Theorem 3.3, there exists a $*$-homomorphism $\psi: C(\mathbf{T}) \rightarrow B$, such that $\psi^{*}=\varphi_{U^{*}}$ and $\psi^{\natural}=\Psi$. $V=\psi(\mathrm{id})$ is the desired unitary.

REMARK 5.2. When, in Theorem 5.1 above, one of the unitaries does not have full spectrum, then condition (1) implies condition (2).

\section{REFERENCES}

1. B. Blackadar, Traces on simple AF $C^{*}$-algebras, J. Funct. Anal. 38 (1980), 156-168.

2. B. Blackadar, O. Bratteli, G. Elliott and A. Kumjian, Reduction of real rank in inductive limits of $C^{*}$-algebras, Math. Ann. 292 (1992), 111-126.

3. B. Blackadar, A. Kumjian and M. Rørdam, Approximately central matrix units and the structure of non-commutative tori, K-Theory 6 (1992), 267-284.

4. B. Blackadar and M. Rørdam, Extending states on preordered semigroups and the existence of quasitraces on $C^{*}$-algebras, J. Algebra 152 (1992), 240-247.

5. J. Cuntz and G. K. Pedersen, Equivalence and Traces on $C^{*}$-algebras, J. Funct. Anal. 33 (1979), 135-164.

6. M. Dardalat and T. Loring, Classifying $C^{*}$-algebras via ordered MOD-p K-theory, Math. Ann. 305 (1996), 601-616.

7. S. Eilers, Kunneth splittings and classification of $C^{*}$-algebras with finitely many ideals, To appear in Fields Comm. Ser. Volume " $C^{*}$-algebras and their applications".

8. G. Elliott, On the classification of $C^{*}$-algebras of real rank zero, J. Reine Angew. Math. 443 (1993), 179-217.

9. G. Elliott, A classification of certain simple $C^{*}$-algebras, Quantum and Non-Commutative Analysis (editors, H. Araki et al.), Kluwer, Dordrecht, (1993), 373-385.

10. G. Elliott, A classification of certain simple $C^{*}$-algebras II, Preprint (1993).

11. G. Elliott, Normal Elements of a simple $C^{*}$-algebra, Algebraic Methods in Operator Theory (R. Curto and P. E. T. Jørgensen, eds.), Birkhäuser, Basel (1994).

12. K. R. Goodearl, Partially Ordered Abelian Groups with Interpolation, Amer. Math. Soc. Providence, Rhode Island, 1986.

13. U. Haagerup, Quasitraces on exact $C^{*}$-algebras are traces, Manuscript (1991).

14. E. Kirchberg, The classification of purely infinite $C^{*}$-algebras using Kasparov's Theory, Preprint (1994).

15. A. J. Lazar, Spaces of affine continuous functions on simplices, Trans. Amer. Math. Soc. 134 (1968), 503-525.

16. K. E. Nielsen and K. Thomsen, Limits of circle algebras, Exposition. Math. 14 (1996), 1756.

17. N. C. Phillips, A classifications theorem for nuclear purely infinite simple $C^{*}$-algebras, Preprint (1995).

18. M. A. Rieffel, The homotopy groups of the unitary groups of non-commutative tori, J. Operator Theory 4 (1991), 237-254.

19. M. Rørdam, A short proof of Elliott's theorem: $\mathcal{O}_{2} \otimes \mathcal{O}_{2} \simeq \mathcal{O}_{2}$, C.R. Math. Rep. Acad. Sci. Canada XVI (1994), 31-36. 
20. K. Thomsen, Traces, unitary characters and crossed products by Z, Publ. RIMS, Kyoto Univ. 31 (1995), 1011-1029.

21. K. Thomsen, Inductive limits of interval algebras: The tracial state space, Amer. J. Math. 116 (1994), 605-620.

22. K. Thomsen, Finite sums and products of commutators in inductive limit $C^{*}$-algebras, Ann. Inst. Fourier, Grenoble 43 (1993), 225-249.

23. J. Villadsen, The range of the Elliott invariant, J. Reine Angew. Math. 462 (1995), 31-55.

MATEMATISK INSTITUT

AARHUS UNIVERSITET

NY MUNKEGADE

8000 AARHUS C

DENMARK

E-mail address: egede@mi.aau.dk 